\title{
ON THE NONNEGATIVITY OF STRINGY HODGE NUMBERS
}

\author{
SEBASTIÁN OLANO
}

\begin{abstract}
We study the nonnegativity of stringy Hodge numbers of a projective variety with Gorenstein canonical singularities, which was conjectured by Batyrev. We prove that the $(p, 1)$ stringy Hodge numbers are nonnegative, and for threefolds we obtain new results about the stringy Hodge diamond, which hold even when the stringy $E$-function is not a polynomial. We also use the Decomposition Theorem and mixed Hodge theory to prove Batyrev's conjecture for a class of fourfolds.
\end{abstract}

\section{A. Introduction}

The purpose of this paper is to give some positive results towards a conjecture of Batyrev about the nonnegativity of stringy Hodge numbers. All varieties considered are over the field of complex numbers.

The stringy $E$-function is a generalization of the $E$-polynomial, or the Hodge-Deligne polynomial, of an algebraic variety. In [Bat98] and [Bat99], Batyrev introduced this notion for varieties with logterminal singularities and for klt pairs. In the case of a projective variety $X$ with at most Gorenstein canonical singularities, the stringy $E$-function is a rational function with integer coefficients (see Section 1). If we write it as $E_{s t}(X)=E_{s t}(X ; u, v)=\sum b_{p, q} u^{p} v^{q}$, the stringy Hodge numbers are defined as:

$$
h_{s t}^{p, q}(X):=(-1)^{p+q} b_{p, q} .
$$

Batyrev made the following basic conjecture in [Bat98, Conjecture 3.10].

Conjecture A (Batyrev). Let $X$ be a projective variety with Gorenstein canonical singularities. Assume that $E_{s t}(X ; u, v)$ is a polynomial. Then all stringy Hodge numbers $h_{s t}^{p, q}(X)$ are nonnegative.

In [Bat98] the numbers $h_{s t}^{p, q}(X)$ are only called stringy Hodge numbers if $E_{s t}(X)$ is a polynomial, and in that case they are similar to the Hodge numbers of a smooth projective variety (see Section 1). Batyrev's motivation comes from mirror symmetry and in many examples of interest in this area, the stringy $E$-function is a polynomial. However, this function is defined on a larger class of varieties and, even if it is not a polynomial, the nonnegativity of $h_{s t}^{p, q}(X)$ represents a basic numerical constraint on the exceptional divisors in a log-resolution of singularities. Hence, the question is naturally of interest to birational geometry as well.

There are several cases in which Conjecture A is known to be true. The first is that of surfaces with canonical singularities. This follows from two facts: stringy Hodge numbers do not change under crepant morphisms as proved by Batyrev (see Theorem 1.5 below), and every surface with canonical singularities admits a crepant resolution. For toric varieties the Conjecture is also 
true. It was shown that the stringy $E$-function is a polynomial in [Bat98], while Conjecture A was proved in [MP05]. Another case is that of varieties with Gorenstein quotient singularities, where Conjecture A is true as shown in [Bat99] for global quotients, and in general in [Yas04]. In the general case it was shown that stringy Hodge numbers are related to Hodge numbers in orbifold cohomology. For $\operatorname{dim} X=3$ and varieties with terminal isolated singularities of dimension 4 and 5 , the answer is also positive. These results hold without the condition of the stringy $E$-function being a polynomial, as was proved in [SV07]. Finally, more examples of classes of isolated singularities where Conjecture A is true can be found in [Sch12].

In general, for a resolution of singularities $f: Y \rightarrow X$,

$$
h_{s t}^{p, 0}(X)=h^{p, 0}(Y) \text {, }
$$

and this number is nonnegative. For the remaining stringy Hodge numbers we do not have such an interpretation, and a deeper discussion is needed. We start by looking at $h_{s t}^{p, 1}(X)$. Given a $\log$-resolution $f: Y \rightarrow X$ with exceptional divisor $D$, these numbers have an easy description:

$$
h_{s t}^{p, 1}(X)=h^{p, 1}(Y)-\sum h^{p-1,0}\left(D_{i}\right)
$$

where the sum is over all the irreducible components $D_{i}$ of $D$. The first result does not require $E_{s t}(X)$ to be a polynomial.

Theorem B. Let $X$ be a projective variety with Gorenstein canonical singularities. Then:

$$
h_{s t}^{p, 1}(X) \geq 0
$$

for all $p$.

It is a quick application of the fact, proved in [GKKP11], that given a resolution of singularities with exceptional set $D$ of a variety with klt singularities, a $p$-form defined outside of $D$ extends across $D$ without acquiring any poles.

If $E_{s t}(X)$ is a polynomial, then it must have degree $2 n$, where $n=\operatorname{dim}(X)$; moreover $h_{s t}^{p, q}(X)=$ $h_{s t}^{n-p, n-q}(X)$ and $h^{p, q}(X)=0$ if $p>n$ or $q>n$ (see Remark 1.4). Therefore, the only non-zero stringy Hodge numbers lie in a Hodge diamond. This symmetry reduces the conjecture to the upper half of the diamond. Thus, if $X$ is a threefold with polynomial stringy $E$-function, it suffices to show that $h_{s t}^{1,1}(X)$ and $h_{s t}^{2,1}(X)$ are nonnegative. As mentioned above, this was shown without assuming that $E_{s t}(X)$ is a polynomial in [SV07]. One consequence of Theorem B is a new proof of this fact.

More can be said about the diamond for threefolds. As stated above, if $X$ has dimension 3 and has polynomial stringy $E$-function, then

$$
h_{s t}^{2,2}(X)=h_{s t}^{1,1}(X) .
$$

If it is not a polynomial, it can still be shown (Proposition 4.5) that:

$$
h_{s t}^{2,2}(X) \geq h_{s t}^{1,1}(X) .
$$


This follows from a new interpretation of $h_{s t}^{2,2}(X)-h_{s t}^{1,1}(X)$ we give, when $X$ is a threefold, in terms of the analytic local defect of a singularity. This notion was introduced by Kawamata [Kaw88] and it plays an important role in the proof of the existence of a $\mathbb{Q}$-factorialization of a threefold with terminal singularities. The divisors with discrepancy 1 over the singular points allowed him to conclude that this process ends. A careful analysis of the proof (see Proposition 4.3) yields the result stated above.

This result is going to be useful in the proof of Theorem E, and this is one of the reasons we are interested in this level of generality. We obtain the following corollary for threefolds.

Corollary C. Let $X$ be a threefold with Gorenstein canonical singularities. Then

$$
h_{s t}^{p, q}(X) \geq 0
$$

if $p+q \leq 4$.

To study other stringy Hodge numbers when the singular locus of $X$ has high codimension, we follow a strategy similar to that of [SV07]. There the authors used the fact that given a logresolution of singularities of a variety with isolated singularities, the restriction map in higher cohomologies from the smooth variety to the exceptional divisor is surjective. This result admits a generalization to varieties with singular locus of higher dimension, which in turn leads to the following:

Theorem D. Let $X$ be a projective variety with at most Gorenstein canonical singularities. Suppose that the singular locus has codimension $c$. Then

$$
h_{s t}^{p, 2}(X) \geq 0
$$

for $p+2 \leq c$.

For instance, this shows that applying Theorem D to varieties with singular locus of dimension 1 , we obtain that as soon as $\operatorname{dim} X \geq 5$ we have

$$
h_{s t}^{2,2}(X) \geq 0 .
$$

However, it does not apply in general to fourfolds. Using Theorem B, the last step in proving Conjecture A when $\operatorname{dim}(X)=4$ is showing the above inequality holds. This is what we focus on next.

We can assume $X$ has terminal singularities (see Remark 1.6) in which case the singular locus has at most dimension 1. Even the case of the product of a threefold with terminal singularities and a smooth curve is not entirely obvious ${ }^{1}$ : one needs Corollary $\mathrm{C}$ in order to check this inequality (see Section 12.1). It can also be seen that the techniques used for proving Theorem $\mathrm{D}$ do not work in general in dimension 4 (see Section 6) and therefore we need a new approach. To this end, the strategy is to compare $h_{s t}^{2,2}(X)$ with $h_{s t}^{2,2}(H)$, for a general hyperplane $H \subseteq X$. We obtain the result under certain conditions, as an application of Corollary $\mathrm{C}$.

\footnotetext{
${ }^{1}$ It is however if the stringy $E$-function of the threefold is a polynomial.
} 
We define the following condition for a log-resolution of singularities $f: Y \rightarrow X$ which is an isomorphism outside of the singular locus of $X$ :

If $D \subseteq Y$ is the exceptional set of $f$, with irreducible components $D=\bigcup D_{i}$,

(*) $\left.\quad f\right|_{D_{i}}$ has connected fibers, and for any irreducible component $B_{i j} \subseteq D_{i} \cap D_{j}$, $\left.f\right|_{B_{i j}}$ has connected fibers.

Theorem E. Let $X$ be a projective variety of dimension 4 with Gorenstein terminal singularities. Suppose there exists a log-resolution of singularities $f: Y \rightarrow X$ which satisfies $(*)$. Then:

$$
h_{\text {st }}^{2,2}(X) \geq 0,
$$

and hence $h_{s t}^{p, q}(X) \geq 0$ for all $p+q \leq 4$. Moreover, if $E_{s t}(X)$ is a polynomial, then Conjecture $A$ holds for $X$.

The Decomposition Theorem (see Theorem 2.6 below) plays a central role in the proof of the theorem. We use the approach of de Cataldo and Migliorini [dCM05], which, besides giving a decomposition of the cohomologies of $Y$ and $D_{i}$, provides many tools for understanding its interaction with the Hodge decomposition. This in turn allow us to get a simplified description of $h_{s t}^{2,2}(X)$ which can be compared to $h_{s t}^{2,2}(H)$, for example, if condition $(*)$ is satisfied.

Finally, in Section 12 we discuss a class of fourfolds with Gorenstein terminal singularities to which we can apply Theorem E. Roughly speaking, these are fourfolds that satisfy a strong equisingularity condition with respect to generic hyperplane sections along their singular locus (see Definition 12.2). In addition, the terminal threefold singularities appearing as such hyperplane sections are required to have a special type of log-resolution (see Definition 12.3), and this class includes:

- $A_{n}$.

- $D_{2 n+1}$.

- $E_{6}$.

A typical example is the fourfold given by

$$
x_{0} x_{1} x_{2}+x_{5} x_{3}^{2}+x_{5} x_{4}^{2}+x_{3}^{3}+x_{4}^{3}=0
$$

in $\mathbb{P}^{5}$, which is equisingular along three copies of $\mathbb{P}^{1}$ given by $\left(x_{i}=x_{j}=x_{3}=x_{4}=0\right)$ for $\{i, j\} \subseteq\{0,1,2\}$, and the singularities of the hyperplane sections are of type $A_{1}$ if $x_{5} \neq 0$. Other examples include extremal contractions of a smooth fourfold of type $(3,1)$ (that is, the exceptional set is a divisor and its image is a curve) with Gorenstein singularities.

If we make some assumptions on the topology of $X$, using the techniques of the proof of Theorem D we obtain the following corollary . It is a consequence of Proposition 6.1.

Corollary F. Let $X$ be a fourfold with at most Gorestein terminal singularities. If $H^{5}(X)=0$, or equivalently $H^{3}\left(X_{\text {reg }}\right)=0$, then

$$
h^{2,2}(X) \geq 0 .
$$

If moreover $E_{s t}(X)$ is a polynomial, Conjecture $A$ is true for $X$. 
Acknowledgements. I am very grateful to my advisor Mihnea Popa for suggesting the problem, and for his constant support during the project. I am also grateful to Mircea Mustaţă, János Kollár, Mark de Cataldo, Sándor Kovács, Yajnaseni Dutta, Akash Sengupta and Lei Wu for interesting discussions.

\section{B. Preliminaries}

1. Stringy Hodge numbers. In this section we give the definition of stringy Hodge numbers, and review some basic results about them. We use the definition given in [Bat98] in the case the variety is projective.

1.1. We say that a variety $X$ has Gorenstein canonical singularities if it is normal, and the following conditions are satisfied: $X$ has singularities of index 1 , which means that $K_{X}$ is Cartier; and given a log-resolution of singularities $f: Y \rightarrow X$, where $D_{1}, \ldots, D_{r}$ are the irreducible components of the exceptional set, if we write

$$
K_{Y}-f^{*}\left(K_{X}\right)=\sum c_{i} D_{i}
$$

then

$$
a\left(D_{i}, X\right):=c_{i} \geq 0
$$

for all $i$. These numbers are integers and are usually referred as the discrepancy of $D_{i}$ with respect to $X$. The two conditions imply that $X$ is Gorenstein, and the second condition defines a variety with canonical singularities.

The following notation is useful: let $I=\{1, \ldots, r\}$, and for any subset $J \subseteq I$ we define

$$
D_{J}=\bigcap_{j \in J} D_{j}
$$

and in case $J=\emptyset$ we put

$$
D_{\emptyset}=Y \text {. }
$$

Definition 1.1. Let $X$ be a projective variety with Gorenstein canonical singularities. The stringy $E$-function is defined as:

$$
E_{s t}(X ; u, v)=\sum_{J \subseteq I} E\left(D_{J} ; u, v\right) \prod_{j \in J} \frac{u v-(u v)^{a_{j}+1}}{(u v)^{a_{j}+1}-1}
$$

where $a_{j}=a\left(D_{j}, X\right)$, and for a smooth projective variety $Z$,

$$
E(Z ; u, v)=\sum(-1)^{p+q} h^{p, q}(Z) u^{p} v^{q}
$$

is the Hodge-Deligne polynomial (or $E$-polynomial). We often write $E_{s t}(X)$ instead of $E_{s t}(X ; u, v)$.

The key result from Batyrev is that the stringy $E$-function does not depend on the resolution as long as the exceptional set is a divisor and its components with nonzero discrepancy are normal crossings [Bat98, Theorem 3.4]. 
1.2. For a projective variety $X$ with Gorenstein canonical singularities we can write:

$$
E_{s t}(X)=\sum b_{p, q} u^{p} v^{q}
$$

with $b_{p, q} \in \mathbb{Z}$, as it is a rational function.

Definition 1.2 (Stringy Hodge numbers). The $(p, q)$-stringy Hodge number is defined as:

$$
h_{s t}^{p, q}(X)=(-1)^{p+q} b_{p, q} \text {. }
$$

Remark 1.3. In the original definition of stringy Hodge numbers given by Batyrev, it was required the stringy $E$-function to be a polynomial, but as stated above, the nonnegativity of the extended version is interesting from the point of view of birational geometry.

Remark 1.4. The following are some basic properties of the stringy E-function and stringy Hodge numbers that were proved by Batyrev [Bat98]:

(1) If $X$ is smooth, then $E_{s t}(X)=E(X)$ and

$$
h_{s t}^{p, q}(X)=h^{p, q}(X) .
$$

(2) The stringy $E$-function is symmetric with respect to its variables:

$$
E_{s t}(X ; u, v)=E_{s t}(X ; v, u),
$$

and so

$$
h_{s t}^{p, q}(X)=h_{s t}^{q, p}(X)
$$

(3) A Poincaré duality kind of result [Bat98, Theorem 3.7]: if $\operatorname{dim} X=n$, then

$$
E_{s t}(X ; u, v)=(u v)^{n} E_{s t}\left(X ; u^{-1}, v^{-1}\right) .
$$

In case $E_{s t}(X)$ is a polynomial, this means it must have degree $2 n$ and

$$
h_{s t}^{p, q}(X)=h_{s t}^{n-p, n-q}(X) .
$$

The following is usually applied to make some simplifications:

Theorem 1.5 ([Bat98, Theorem 3.12]). Assume that an algebraic variety with Gorenstein canonical singularities $X$ admits a projective birational morphism $f: Y \rightarrow X$ such that $f^{*} K_{X}=K_{Y}$. Then $E_{s t}(X)=E_{s t}(Y)$. In particular the stringy Hodge numbers are the same.

Remark 1.6. For a variety $X$ with canonical singularities, there exists a crepant birational morphism $f: Y \rightarrow X$ such that $Y$ has at most terminal singularities [BCHM10, Corollary 1.4.3]. This was proved in dimension 3 by Reid [Rei83, Main Theorem]. Using Theorem 1.5 we can assume that $X$ has at most terminal singularities.

1.3. The following is useful for simplifying various expressions.

Notation. Let $f: Y \rightarrow X$ be a log-resolution of singularities of $X$ and $\bigcup_{i \in I} D_{i}=D \subseteq Y$ be the reduced exceptional divisor. We denote:

$$
D(p):=\coprod_{\substack{J \subseteq I \\|J|=p}} D_{J} .
$$


Remark 1.7. Let $X$ be a projective variety with at most Gorenstein terminal singularities. The following are explicit descriptions of some stringy Hodge numbers:

- $h_{s t}^{p, 0}(X)=h^{p, 0}(Y)$.

- $h_{s t}^{p, 1}(X)=h^{p, 1}(Y)-h^{p-1,0}(D(1))$.

- $h_{s t}^{p, 2}(X)=h^{p, 2}(Y)-h^{p-1,1}(D(1))+h^{p-2,0}(D(2))+\sum_{a_{j}=1} h^{p-2,0}\left(D_{j}\right)$

where $a_{j}=a\left(D_{j}, X\right)$.

Note that as $D(p)$ is a disjoint union of smooth projective varieties, its cohomology spaces are direct sums of the cohomologies of its components. For example $H^{k}(D(1))=\bigoplus H^{k}\left(D_{j}\right)$.

Notation. There is always a piece of the summands of stringy Hodge numbers in which discrepancies do not show up. That is,

$$
h_{s t}^{p, q}(X)=\sum(-1)^{k} h^{p-k, q-k}(D(k))+\text { extra terms, }
$$

so it is convenient to define

$$
a_{p, q}(X):=\sum(-1)^{k} h^{p-k, q-k}(D(k)) .
$$

2. Background results in mixed Hodge theory and perverse sheaves. This section contains most of the known facts that are used later in the paper, for easy reference.

2.1. We start with some results about the logarithmic complex; see e.g. [Voi07, Section 8]. They are used in the proof of Theorem B keeping the same notation.

Let $Y$ be a smooth projective variety and $D$ a simple normal crossings divisor in $Y$.

Definition 2.1. Let $\Omega_{Y}^{p}(\log D)$ be the sheaf of $p$-forms with logarithmic poles along $D$. The increasing weight filtration consists of subsheaves

$$
W_{k} \Omega_{Y}^{p}(\log D) \subseteq \Omega_{Y}^{p}(\log D)
$$

such that if $z_{1}, \ldots, z_{n}$ are local coordinates on an open set $V$, and $D$ is given by the equation

$$
z_{1} \cdots z_{r}=0
$$

then $\left.W_{k} \Omega^{p}(\log D)\right|_{W}$ is a free $\mathscr{O}_{W}$ module generated by elements of the form

$$
\frac{d z_{i_{1}}}{z_{i_{1}}} \wedge \cdots \wedge \frac{d z_{i_{s}}}{z_{i_{s}}} \wedge d z_{j_{1}} \wedge \cdots \wedge d z_{j_{p-s}}
$$

with $i_{l} \leq r$ and $s \leq k$.

The sheaves of logarithmic poles form the logarithmic sequence $\Omega_{Y}^{\bullet}(\log D)$, and one crucial result is the isomorphism

$$
H^{k}(Y \backslash D, \mathbb{C}) \cong \mathbb{H}^{k}\left(Y, \Omega_{Y}^{\bullet}(\log D)\right)
$$

due to Deligne [Del71, Proposition 3.1.8]. 
The weight filtration on the sheaves induces one on the sequence, which in turn defines a weight filtration on the cohomology of $Y \backslash D$, and it is the same as the one discussed in Section 2.2.

Using the same notation as in Section 1.3 and letting $j_{k}: D(k) \hookrightarrow Y$ we have the following (see e.g. [Voi07, Proposition 8.32]):

Proposition 2.3. There exists a natural isomorphism

$$
W_{k} \Omega_{Y}^{p}(\log D) / W_{k-1} \Omega_{Y}^{p}(\log D) \cong j_{k *} \Omega_{D(k)}^{p-k} .
$$

2.2. The cohomology spaces of an algebraic variety are endowed with a mixed Hodge structure functorial with respect to algebraic morphisms, as proved by Deligne. We state a few facts used in several parts of this paper (see e.g. [CEZGL14, Section 3]).

Let $X$ be an algebraic variety of dimension $n$. For simplicity all cohomologies are assumed to be with complex coefficients. There exists an increasing filtration

$$
\{0\}=W_{-1} \subseteq W_{0} \subseteq \cdots \subseteq W_{2 k}=H^{k}(X)
$$

of vector subspaces called the weight filtration. The graded pieces are defined to be

$$
\mathrm{Gr}_{j}^{W} H^{k}(X)=W_{j} / W_{j-1}
$$

There also exists a decreasing filtration

$$
\{0\}=F^{m} \subseteq F^{m-1} \subseteq \cdots \subseteq F^{0}=H^{k}(X)
$$

of vector subspaces, called the Hodge filtration, which induces a pure Hodge structure of weight $j$ on $\operatorname{Gr}_{j}^{W} H^{k}(X)$.

Remark 2.4. The following are basic facts about the weights of the cohomology $H^{k}(X)$ :

(i) Suppose $X$ is a smooth variety. Then $W_{k-1}=0$, that is, it only has the "upper weights".

(ii) Suppose $X$ is a proper variety. Then $W_{k}=H^{k}(X)$, that is, it only has the "lower weights".

2.3. The mixed Hodge structure of a simple normal crossings variety can be described in a simple way. This computation is used, for example, in the proof of Theorem D. See e.g. [Elz83, Part II, 1] and [CEZGL14, Section 3].

Let $D$ be a simple normal crossings variety and $H^{k}(D(r)) \stackrel{\delta_{r}}{\longrightarrow} H^{k}(D(r+1))$ be the alternating sum of the pullbacks of the natural inclusions $D(r+1) \hookrightarrow D(r)$. We get the complex

$$
0 \longrightarrow H^{k}(D(1)) \stackrel{\delta_{1}}{\longrightarrow} H^{k}(D(2)) \stackrel{\delta_{2}}{\longrightarrow} \cdots \stackrel{\delta_{l}}{\longrightarrow} H^{k}(D(l+1)) \stackrel{\delta_{l+1}}{\longrightarrow} \cdots,
$$

in which all cohomologies have $\mathbb{C}$-coefficients. The weight $k$ piece of the mixed Hodge structure on the cohomology of $D$ are the cohomologies of this complex. More precisely, we have

$$
\operatorname{Gr}_{k}^{W} H^{k+l}(D)=\operatorname{ker} \delta_{l+1} / \operatorname{im} \delta_{l} \text {. }
$$

The Hodge space $H^{p, q}\left(\operatorname{Gr}_{k}^{W} H^{k+l}(D)\right)$ is obtained by applying first $H^{p, q}$ to the complex and then taking the cohomologies. 
2.4. The Decomposition Theorem and related results are used in the proof of Theorem E. We gather them following the notation of de Cataldo and Migliorini [dCM05].

For an algebraic variety $X$ we denote $\mathrm{D}(X)$ the category of constructible complexes, and by $\operatorname{Perv}(X)$ the abelian subcategory of perverse sheaves, together with the perverse cohomology functors

$$
{ }^{\mathfrak{p}} \mathcal{H}^{k}: \mathrm{D}(X) \rightarrow \operatorname{Perv}(X) .
$$

The simple objects of $\operatorname{Perv}(X)$ are the intersection cohomology complexes $I C_{X}(L)$, where $L$ is a local system in $U \subseteq X_{\text {reg }}$. An explicit definition can be found in [HTT08, Proposition 8.2.11]. When the local system $L=\mathbb{C}_{U}$, we simply denote the intersection cohomology complex as $I C_{X}$.

Theorem 2.6 (Decomposition theorem). Let $f: Y \rightarrow X$ be a projective morphism from a smooth variety $Y$ of dimension $n$. Then:

$$
\mathbf{R} f_{*} \mathbb{C}_{Y}[n] \cong \bigoplus^{\mathfrak{p}} \mathcal{H}^{k}\left(\mathbf{R} f_{*} \mathbb{C}_{Y}[n]\right)[-k]
$$

in $\mathrm{D}(X)$. Moreover, each ${ }^{\mathfrak{p}} \mathcal{H}^{k}\left(\mathbf{R} f_{*} \mathbb{C}_{Y}[n]\right)$ can be decomposed into intersection complexes of the strata.

A direct consequence of the theorem is that we get a direct sum decomposition of the cohomology spaces of $Y$, by taking hypercohomology functors. More will be said about this decomposition in Section 2.5.

The next results are used when comparing the direct sum decomposition of the cohomologies of $Y$ to the one we get on certain subvarieties, by applying the theorem to the restriction map. They work in general for a normally nonsingular inclusion, but its definition is rather technical and can be found in [dCM05, Section 3.5]. Good examples are subvarieties of the ambient projective space of a projective variety, intersecting all of the strata (of a given stratification) transversely. The example we are interested in is a general hyperplane $H \subseteq X$.

Lemma 2.7 ([dCM05, Lemma 4.3.8]). Let

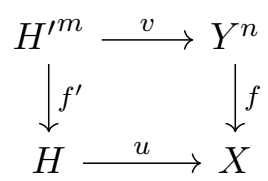

be a Cartesian diagram of maps of algebraic varieties of the indicated dimensions and $f$ proper. Assume that $u$ is a normally nonsingular inclusion. Then

$$
{ }^{\mathfrak{p}} \mathcal{H}^{i}\left(\mathbf{R} f_{*}^{\prime} \mathbb{C}_{H^{\prime}}[m]\right) \cong u^{* \mathfrak{p}} \mathcal{H}^{i}\left(\mathbf{R} f_{*} \mathbb{C}_{Y}[n]\right)[m-n]
$$

for every $i \in \mathbb{Z}$ and the natural map

$$
v^{*}: H^{k}(Y) \rightarrow H^{k}\left(H^{\prime}\right)
$$

is compatible with the direct sum decomposition in perverse cohomology groups and it is strict. 
Proposition 2.9 ([dCM05, Proposition 4.7.7]). Let $P \in \operatorname{Perv}(X)$. Then the natural map

$$
u^{*}: \mathbb{H}^{j}(X, P) \rightarrow \mathbb{H}^{j}\left(H, u^{*} P\right)
$$

is an isomorphism for $j \leq-2$ and injective for $j=1$.

2.5. We now consider perverse cohomology groups.

Definition 2.10. Using the same notation as in Theorem 2.6 we define the subspaces:

$$
H_{b}^{n+l}(Y):=\mathbb{H}^{l-b}\left(X,{ }^{\mathfrak{p}} \mathcal{H}^{b}\left(\mathbf{R} f_{*} \mathbb{C}_{Y}[n]\right)\right) .
$$

We have a direct sum decomposition

$$
H^{k}(Y)=\bigoplus H_{b}^{k}(Y)
$$

which can be made into a decomposition by Hodge substructures [dCM05, Remark 2.1.6]. Understanding these spaces is the main task in the proof of Theorem E and one of the tools we use is:

Theorem 2.11 (Global invariant cycle theorem). Suppose $f: Z \rightarrow U$ is a smooth projective map and let $\bar{Z}$ be a smooth compactification of $Z$. Then for $x \in U$,

$$
H^{0}\left(U, R^{k} f_{*} \mathbb{C}_{Z}\right)=H^{k}\left(f^{-1}(x)\right)^{\pi_{1}(U, x)}=\operatorname{im}\left\{H^{k}(\bar{Z}) \rightarrow H^{k}\left(f^{-1}(x)\right)\right\} .
$$

Finally, we have the following analogues of classical results in Hodge theory [dCM05, Theorem 2.1.4, Theorem 2.2.3]:

Theorem 2.12 (Hard Lefschetz Theorem for Perverse cohomology groups). Let $k \geq 0$ and $b, j \in \mathbb{Z}$. Then the following cup product maps are isomorphisms:

(i) $\eta^{k}: H_{-k}^{j}(Y) \cong H_{k}^{j+2 k}(Y)$ where $\eta \in H^{2}(Y)$ is an ample class.

(ii) $L^{k}: H_{b}^{n+b-k}(Y) \cong H_{b}^{n+b+k}(Y)$ where $L=f^{*} A \in H^{2}(Y)$ for an ample class $A \in H^{2}(X)$.

Theorem 2.13 (Weak Lefschetz Theorem for Intersection Cohomology). Let $u: H \hookrightarrow X$ be $a$ general hyperplane section of a projective variety. Then

$$
u^{*}: I H^{j}(X) \rightarrow I H^{j}(H)
$$

is an isomorphism for $j \leq \operatorname{dim} X-2$ and injective for $j=\operatorname{dim} X-1$.

Theorem 2.14 (Hard Lefschetz Theorem for Intersection Cohomology). Let $X$ be a projective variety. There is an isomorphism

$$
A^{j}: I H^{\operatorname{dim} X-j}(X) \rightarrow I H^{\operatorname{dim} X+j}(X)
$$

where $A$ is an ample class in $X$. 


\section{First Results}

3. ( $p, 1)$-stringy Hodge numbers. In this section we prove Theorem B. The result is an application of Theorem 2.3 and Proposition 3.3. Recall that for a projective variety with at most Gorenstein canonical singularities, the $(p, 1)$-stringy Hodge number is defined as:

$$
h_{s t}^{p, 1}(X)=h^{p, 1}(Y)-h^{p-1,0}(D(1))
$$

where $f: Y \rightarrow X$ is a log-resolution of singularities and $D$ the exceptional set.

We use the notation in Section 2.1. By Proposition 2.3, we have the following exact sequence:

$$
0 \rightarrow \Omega_{Y}^{p} \rightarrow W_{1} \Omega_{Y}^{p}(\log D) \rightarrow \bigoplus \Omega_{D_{i}}^{p-1} \rightarrow 0
$$

Consider the long exact sequence of cohomologies:

$$
0 \rightarrow H^{0}\left(\Omega_{X}^{p}\right) \rightarrow H^{0}\left(W_{1} \Omega_{X}^{p}(\log D)\right) \rightarrow \bigoplus H^{0}\left(\Omega_{D_{i}}^{p-1}\right) \stackrel{d^{\prime}}{\rightarrow} H^{1}\left(\Omega_{X}^{p}\right) \rightarrow \ldots
$$

We use the following simplified version of [GKKP11, Proposition 19.1].

Proposition 3.3. Let $X$ be a complex quasi-projective variety with at most canonical singularities, and $f: Y \rightarrow X$ a log-resolution with exceptional set $D \subseteq Y$. Then the natural injection

$$
H^{0}\left(Y, \Omega_{Y}^{p}\right) \rightarrow H^{0}\left(Y, \Omega_{Y}^{p}(\log D)\right)
$$

is an isomorphism.

Proof of Theorem B. The inclusion $H^{0}\left(\Omega_{Y}^{p}\right) \hookrightarrow H^{0}\left(W_{1} \Omega_{Y}^{p}(\log D)\right)$ is actually an isomorphism by Proposition 3.3. Indeed, $H^{0}\left(\Omega_{Y}^{p}\right) \hookrightarrow H^{0}\left(W_{1} \Omega_{Y}^{p}(\log D)\right)$ factors through $H^{0}\left(\Omega_{Y}^{p}\right) \hookrightarrow H^{0}\left(\Omega_{Y}^{p}(\log D)\right)$ which is an isomorphism. Hence, $d^{\prime}$ in (3.2) is injective. Taking dimensions we get:

$$
h^{p, 1}(Y)-h^{p-1,0}(D(1)) \geq 0
$$

which is precisely the statement

$$
h_{s t}^{p, 1}(X) \geq 0
$$

This result, together with the description of $h_{s t}^{p, 0}(X)$ (see Section 1.7), implies the following corollary. It was originally proved in [SV07] with a different method.

Corollary 3.4. If $X$ is a projective threefold with Gorenstein canonical singularities, then

$$
h_{s t}^{p, q}(X) \geq 0
$$

for $p+q \leq 3$. In particular, Conjecture $A$ holds for $\operatorname{dim} X=3$. 
4. Threefolds. In this section we prove Proposition 4.5 which is used in the proof of Theorem E, and expands the information about the stringy Hodge diamond when $\operatorname{dim} X=3$.

Let $X$ be a projective threefold with at most Gorenstein terminal singularities. It is enough to consider this case by Remark 1.6. If $E_{s t}(X)$ is a polynomial, one of the consequences is that

$$
h_{s t}^{2,2}(X)=h_{s t}^{1,1}(X)
$$

by Remark 1.4(3). As it was shown that $h_{s t}^{1,1}(X) \geq 0$, the same is true for $h_{s t}^{2,2}(X)$. If $E_{s t}(X)$ is not a polynomial, then few things are known about the lower stringy Hodge triangle. For example, some numbers might fail to be nonnegative; see the example of a threefold with $h_{s t}^{3,3}(X)<0$ in [SV07, Remark 3.5(1)].

We give a new interpretation of the number $h_{s t}^{2,2}(X)-h_{s t}^{1,1}(X)$ in terms of the analytic local defect, and use the existence of an analytic $\mathbb{Q}$-factorialization of a threefold terminal singularity in [Kaw88], to show that

$$
h_{s t}^{2,2}(X) \geq 0 .
$$

Definition 4.1. Let $H$ be a threefold with isolated singularities. For a point $x \in H$ we define the local defect of $H$ at $x$ as

$$
\sigma(H, x)=\operatorname{dim} \operatorname{Weil}\left(U_{x}\right)_{\mathbb{Q}} / \operatorname{Cart}\left(U_{x}\right)_{\mathbb{Q}}
$$

where $U_{x}$ is a contractible Stein open neighborhood of $x$ in $H$. If $\sigma(H, x)=0$, we say that $H$ is analytically $\mathbb{Q}$-factorial at $x$.

If $H$ has rational singularities, the local defect is finite at every point [Kaw88, Lemma 1.12]. Using the strategy of the proof of this fact we can get a convenient description of $\sigma(H, x)$. Let $\left\{x_{1}, \ldots, x_{m}\right\}$ be the isolated singularities of $H$, and $x_{i} \in U_{i}$ be a Stein contractible neighborhood. For a $\log$-resolution $f: H^{\prime} \rightarrow H$, we have isomorphisms

$$
\operatorname{Div}^{0}\left(U_{i}\right) \cong \operatorname{Div}^{0}\left(V_{i}\right)
$$

where $V_{i}=f^{-1}\left(U_{i}\right)$. As $U_{i}$ is Stein, we can apply Theorem B of Cartan [Car53], and obtain that $\operatorname{Pic}\left(U_{i}\right)=0$. Let $f^{-1}\left(x_{i}\right)=\bigcup E_{i, j}$. Using the sequence

$$
\mathbb{Z}\left[E_{i, j}\right] \rightarrow \operatorname{Weil}\left(V_{i}\right) \rightarrow \operatorname{Weil}\left(V_{i} \backslash E_{i}\right) \rightarrow 0,
$$

and the fact that

$$
\operatorname{Weil}\left(V_{i} \backslash E_{i}\right) \cong \operatorname{Weil}\left(U_{i}\right),
$$

we get the following isomorphism given in [NS95, 3.9]:

$$
\operatorname{Weil}\left(U_{i}\right) / \operatorname{Cart}\left(U_{i}\right) \cong H^{1}\left(V_{i}, O_{V_{i}}^{*}\right) / \sum \mathbb{Z}\left[E_{i, j}\right]
$$

Using again the fact that $H$ has rational singularities, we conclude that

$$
H^{1}\left(V_{i}, O_{V_{i}}^{*}\right) \cong H^{2}\left(E_{i}, \mathbb{Z}\right)
$$

Therefore, we have the following expression in terms of the cohomology of $E_{i}$ :

$$
\sigma\left(H, x_{i}\right)=\operatorname{dim} H^{2}\left(E_{i}\right) / \sum \mathbb{C}\left[E_{i, j}\right]
$$


Proposition 4.3. Let $H$ be a threefold with Gorenstein terminal singularities and $x \in H$ a singular point. Then

$$
\sigma(H, x) \leq \#\left\{E_{j}: a\left(E_{j}, H\right)=1 \text { and } \operatorname{center}_{H}\left(E_{j}\right)=x\right\} .
$$

Proof. We start with the same set up as in the proof of [Kaw88, Corollary 4.5]. Let $V=V_{0}$ be a Stein contractible neighborhood of $x$. Suppose it is not $\mathbb{Q}$-factorial and let $D_{0} \in \operatorname{Weil}\left(V_{0}\right)$ which is not $\mathbb{Q}$-Cartier. There exists an analytic space and a projective morphism $f_{0}: V_{1} \rightarrow V_{0}$ which is an isomorphism in codimension 1 and the strict transform of $D_{0}$ is $\mathbb{Q}$-Cartier [Kaw88, Theorem 4.1 and Lemma 3.1]. $V_{1}$ has also terminal singularities and $\operatorname{Pic}\left(V_{1}\right) \cong H^{2}\left(V_{1}, \mathbb{Z}\right)$. We get

$$
\sigma\left(V_{0}, x\right)=\sigma\left(V_{1}, f_{0}^{-1}(x)\right)+b_{2}\left(V_{1}\right)
$$

where $\sigma\left(V_{1}, f_{0}^{-1}(x)\right)=\operatorname{dim} \operatorname{Weil}\left(V_{1}\right)_{\mathbb{Q}} / \operatorname{Cart}\left(V_{1}\right)_{\mathbb{Q}}$. $V_{1}$ retracts to the exceptional set of $f_{0}$ which consists of a union of curves. The blow up of the any of these curves (which are smooth in $V_{1}$ ) produce a discrepancy 1 divisor over $x$ [KM98, Proof of Theorem 6.25], and each of them have a different center in $V_{1}$. The conclusion is that the local defect and the number of discrepancy 1 divisors over $x$ drop by the same number.

We continue the process by induction. Suppose $V_{n}$ is not $\mathbb{Q}$-factorial and let $D_{n} \in$ Weil $\left(V_{n}\right)$ which is not $\mathbb{Q}$-Cartier. As before, there exists a projective morphism $f_{n}: V_{n+1} \rightarrow V_{n}$ with the same conditions as $f_{0}$ and $D_{0}$, in particular the exceptional set is a union of curves. Let $g_{n}: V_{n} \rightarrow V_{0}$ be the composition of the previous morphisms. The exceptional set is also a union of curves, and $V_{n}$ retract onto them. As $f_{n}$ is an isomorphism outside of the isolated singularities of $V_{n}$, the class of each of the curves $C_{i}$ in $H^{2}\left(V_{n}, \mathbb{Z}\right)$ maps to the class of the curve $f_{n *}^{-1} C_{i}$ in $H^{2}\left(V_{n+1}, \mathbb{Z}\right)$. Therefore,

$$
\sigma\left(V_{n}, g_{n}^{-1}(x)\right)=\sigma\left(V_{n+1}, g_{n+1}^{-1}(x)\right)+b_{2}\left(V_{n+1}\right)-b_{2}\left(V_{n}\right),
$$

that is, it drops by the number of exceptional curves of $f_{n}$. They also correspond to discrepancy 1 divisors over $x$ with different centers in $V_{n+1}$. So at each step, the local defect is dropping by the number of discrepancy 1 divisors over $x$ that come from blowing up the exceptional curves of the map.

The process stops and we reach an analytic space $W$ which is $\mathbb{Q}$-factorial. For $W$ the claim is trivial as the local defect is 0 . The result follows.

Every irreducible component $E_{i, j}$ of an exceptional divisor over a terminal singularity of a threefold is birational to a ruled surface [Rei80, Corollary 2.14]. This implies $b_{2}\left(E_{i, j}\right)=h^{1,1}\left(E_{i, j}\right)$, as $h^{2,0}$ is a birational invariant.

The vector space $H^{2}\left(E_{i}\right)$ has a pure Hodge structure (cf. proof of Proposition 2.1 in [NS95]), which implies that

$$
b_{2}\left(E_{i}\right)=b_{2}\left(E_{i}(1)\right)-b_{2}\left(E_{i}(2)\right)
$$

(see Section 2.3), and therefore

$$
b_{2}\left(E_{i}\right)=h^{1,1}\left(E_{i}(1)\right)-h^{0}(E(2)) .
$$


We also have an inclusion

$$
H_{4}\left(E_{i}\right) \hookrightarrow H^{2}\left(E_{i}\right),
$$

which means the classes $\mathbb{C}\left[E_{i, j}\right]$ are independent in $H^{2}\left(E_{i}\right)$ (see for example [Ste83, Corollary 1.12]). Plugging in this information into (4.2) we get:

$$
\sigma\left(H, x_{i}\right)=h^{1,1}\left(E_{i}(1)\right)-h^{0}\left(E_{i}(2)\right)-h^{0}\left(E_{i}(1)\right) .
$$

Proposition 4.5. For a projective threefold $H$ with at most Gorenstein canonical singularities

$$
h_{s t}^{2,2}(H) \geq h_{s t}^{1,1}(H) .
$$

Proof. We can assume $H$ has terminal singularities by Remark 1.6. Let $f^{\prime}: H^{\prime} \rightarrow H$ be a logresolution with exceptional set $E=\bigcup E_{i, j}$. Recall the description of the stringy Hodge numbers in Remark 1.7. We have:

$$
h_{s t}^{2,2}(H)-h_{s t}^{1,1}(H)=-h^{1,1}(E(1))+h^{0}(E(2))+h^{0}(E(1))+\sum_{a_{i j}=1} h^{0}\left(E_{i, j}\right) .
$$

Note that all exceptional divisors of discrepancy 1 must show up in $f^{\prime}$ [KM98, Proof of Lemma 6.36]. By taking addition over every singularity, Proposition 4.3 together with (4.4) is equivalent to

$$
h_{s t}^{2,2}(H)-h_{s t}^{1,1}(H) \geq 0 .
$$

Proof of Corollary C. This is a consequence of Theorem B and Proposition 4.5.

5. Low dimensional singular locus. In this section we prove Theorem $\mathrm{D}$. We in fact prove a more general result. Recall that $h_{s t}^{p, q}(X)$ can be written as the sum of two pieces: one (called $a_{p, q}(X)$ in (1.8)) which does not depend on the discrepancy of the irreducible components of the exceptional divisor, and another one which does. We show that $a_{p, q}(X)$ is nonnegative under some conditions of the dimension of the singular locus.

If a variety has isolated singularities, the higher cohomologies of the exceptional divisor have a pure Hodge structure [Ste83, Corollary 1.12]. The following is a generalization without restriction on the dimension of the singular locus:

Theorem 5.1 ([PS08, Theorem 6.31]). Let $X$ be an algebraic variety of dimension $n$. Let $Z$ be the singular locus which has dimension $s$ and $f: Y \rightarrow X$ a resolution such that $f^{-1}(Z)=D$ is a simple normal crossings divisor on $Y$. Then:

- $W_{k-1} H^{k}(D)=0$ for all $k \geq n+s$.

- If $Z$ is compact, then $H^{k}(D)$ is pure of weight $k$ for all $k \geq n+s$.

We reproduce the proof from [PS08], as we need to use some of its intermediate steps in the proof of Proposition 5.3.

Proof. Let $U_{0}, \ldots, U_{s}$ be $(s+1)$ affine open subsets of $X$ which cover $Z$. In the projective case we can take $U_{i}$ to be the complement of a general ample divisor. Indeed, as the intersection of these divisors is a variety of codimension $s$, and the divisors are general, this subvariety does not intersect $Z$, and hence $U:=U_{0} \cup \ldots \cup U_{s}$ covers $Z$. As $U_{i}$ is affine, it is homotopic to a CW-complex of 
dimension $n$, and then $H^{k}\left(U_{i}\right)=0$ for $k>n$; see e.g. [GM88, Part II, 5.1*]. Using Mayer-Vietoris and induction we get that

$$
H^{k}(U)=0
$$

for $k>n+s$.

Let $\tilde{U}=f^{-1}(U)$. For the birational morphism $\left.f\right|_{\tilde{U}}$ we have the following long exact sequence:

$$
\ldots \rightarrow H^{k}(U) \rightarrow H^{k}(\tilde{U}) \oplus H^{k}(Z) \rightarrow H^{k}(D) \rightarrow H^{k+1}(U) \rightarrow \ldots
$$

(see for instance [PS08, Corollary-Definition 5.37]). For $k \geq n+s$ we get a surjection

$$
H^{k}(\tilde{U}) \rightarrow H^{k}(D) \text {. }
$$

As $\tilde{U}$ is smooth, it only has the upper weights (Remark 2.4(i)). From this we get the first result. If moreover $Z$ is compact, then $D$ is compact as well, and hence it only has lower weights (Remark 2.4(ii)). The result follows.

Proposition 5.3. Let $X$ be a projective variety of dimension $n$ with Gorenstein canonical singularities. Let $Z$ be its singular locus and suppose it has dimension $s$. Then $a_{p, q}(X)$ is nonnegative for all $p, q$ such that $p+q \leq n-s$.

Proof. Let $f: Y \rightarrow X$ be a log-resolution and $f^{-1}(Z)=D$ a simple normal crossings divisor. Let $k:=2 n-p-q$. Note that by assumption we have that $k \geq n+s$. By Theorem 5.1 we get that $H^{k}(D)$ has a pure Hodge structure. The complex used to describe the mixed Hodge structure of $H^{k}(D)$ (see Section 2.3) is then an exact sequence:

$$
0 \rightarrow H^{k}(D) \rightarrow H^{k}(D(1)) \rightarrow \ldots \rightarrow H^{k}(D(p)) \rightarrow 0 .
$$

Taking the $h^{n-p, n-q}$ pieces we get

$$
h^{p, q}(D)=\sum_{i \geq 1}(-1)^{i+1} h^{n-p, n-q}(D(i))=\sum_{i \geq 1}(-1)^{i+1} h^{p-i, q-i}(D(i)) .
$$

Let $U$ be as in the proof of Theorem 5.1. We have a surjection $H^{k}(\tilde{U}) \rightarrow H^{k}(D)$. As $Y$ is a smooth compactification of $\tilde{U}$, we have that the image of $H^{k}(Y) \rightarrow H^{k}(D)$ is the same as that of $H^{k}(\tilde{U}) \rightarrow H^{k}(D)$ [Del74, Prop. 8.2.6]. In particular, we obtain that

$$
h^{p, q}(Y) \geq h^{p, q}(D)=\sum_{i \geq 1}(-1)^{i+1} h^{p-i, q-i}(D(i)),
$$

and therefore

$$
a_{p, q}(X)=\sum_{i \geq 0}(-1)^{i} h^{p-i, q-i}(D(i)) \geq 0
$$

Proof of Theorem D. This is a direct consequence of Proposition 5.3. Recall that

$$
h_{s t}^{p, 2}(X)=a_{p, 2}(X)+\sum_{a_{j}=1} h^{0}\left(D_{j}\right)
$$


Since $a_{p, 2}(X) \geq 0$, we have

$$
h_{s t}^{p, 2}(X) \geq 0
$$

\section{MAIN THEOREM}

6. Fourfolds. A consequence of Theorem B applied to a projective fourfold $X$ with Gorenstein terminal singularities, together with the fact that $h_{s t}^{p, 0}(X) \geq 0$ always holds (see Remark 1.7), is that

$$
h_{s t}^{p, q}(X) \geq 0
$$

when $p+q \leq 4$ and $(p, q) \neq(2,2)$. This means that for Conjecture A to hold, it remains to prove $h_{s t}^{2,2}(X) \geq 0$. If $X$ has isolated singularities, Theorem D implies the inequality above. Therefore, for the rest of the paper we discuss fourfolds whose singular locus has at least one component of dimension 1.

Let $f: Y \rightarrow X$ be a log-resolution of singularities, $D$ the exceptional set, and $C$ the singular locus of $X$. Recall that

$$
h_{s t}^{2,2}(X)=a_{2,2}(X)+\sum_{a_{j}=1} h^{0}\left(D_{j}\right)
$$

and

$$
a_{2,2}(X)=h^{2,2}(Y)-h^{1,1}(D(1))+h^{0}(D(2)) .
$$

Using the isomorphism (2.2), and denoting $U:=Y \backslash D \cong X_{\text {reg }}$, we have

$$
a_{2,2}(X)=h^{2,2}\left(\operatorname{Gr}_{4}^{W} H^{4}(U)\right)-h^{2,2}\left(\operatorname{Gr}_{4}^{W} H^{3}(U)\right)+h^{2,2}\left(\operatorname{Gr}_{4}^{W} H^{2}(U)\right)^{2} .
$$

Indeed, the complex $E_{1}^{*, 4}$ given by the spectral sequence used to compute the weight filtration on the cohomologies of $U$ is precisely

$$
0 \rightarrow H^{0}(D(2)) \rightarrow H^{2}(D(1)) \rightarrow H^{4}(Y) \rightarrow 0,
$$

where the maps are alternating sums of the Gysin morphisms (see [Voi07, Corollary 8.33 and Proposition 8.34]). These are maps of Hodge structures, and the spectral sequence degenerates at $E_{2}$ with $E_{2}^{p, q}=\operatorname{Gr}_{q}^{W} H^{p+q}(U)$ (see [Voi07, Theorem 8.35]). The equality above follows by taking the corresponding Hodge pieces.

A sufficient condition for $a_{2,2}(X)$ to be nonnegative, and as a consequence $h_{s t}^{2,2}(X)$ as well, is

$$
h^{2,2}\left(\operatorname{Gr}_{4}^{W} H^{3}(U)\right)=0 .
$$

This is equivalent by Poincaré duality to

$$
h^{2,2}\left(\mathrm{Gr}_{4}^{W} H_{c}^{5}(U)\right)=0 .
$$

On the other hand, using the sequence of the pair $(X, C)$ we obtain

$$
H_{c}^{5}(U) \cong H^{5}(X)
$$

\footnotetext{
${ }^{2}$ This last term $h^{2,2}\left(\operatorname{Gr}_{4}^{W} H^{2}(U)\right)$ can actually be shown to be equal to 0 , but this does not affect the discussion below.
} 
as mixed Hodge structures. This means that an equivalent sufficient condition is $h^{2,2}\left(\mathrm{Gr}_{4}^{W} H^{5}(X)\right)=$ 0 . With this discussion we get the following.

Proposition 6.1. Let $X$ be a fourfold with at most Gorenstein terminal singularities. If $W_{4} H^{5}(X)=$ 0 , then

$$
h_{s t}^{2,2}(X) \geq 0 .
$$

If moreover $E_{s t}(X)$ is a polynomial, Conjecture $A$ is true for $X$. In particular, if $H^{5}(X)=0$, or equivalently $H^{3}\left(X_{\text {reg }}\right)=0$, all of the above holds.

The following example shows that $a_{2,2}(X)$ can be negative, and therefore arguments to prove that in general $h_{s t}^{2,2}(X) \geq 0$ must take into consideration the term $\sum_{a_{j}=1} h^{0}\left(D_{j}\right)$.

Example 6.2. Let $X_{0}$ be the Burkhardt quartic, given by the equation

$$
x_{0}^{4}-x_{0}\left(x_{1}^{3}+x_{2}^{3}+x_{3}^{3}+x_{4}^{3}\right)+3 x_{1} x_{2} x_{3} x_{4}=0
$$

in $\mathbb{P}^{4}$. This threefold has 45 nodes, and blowing them up we get $Y_{0}$ which is a smooth rational variety [HW01, Corollary 2.5] with $b_{2}\left(Y_{0}\right)=61$ [HW01, Corollary 2.12]. As $Y_{0}$ is rational,

$$
h^{2,0}\left(Y_{0}\right)=0,
$$

hence

$$
h^{2,2}\left(Y_{0}\right)=h^{1,1}\left(Y_{0}\right)=61 .
$$

Moreover, the exceptional divisor over every node is isomorphic to $\mathbb{P}^{1} \times \mathbb{P}^{1}$. Let $X=X_{0} \times \mathbb{P}^{1}$. As $X_{0}$ only has nodes as singularities, $E_{s t}\left(X_{0}\right)$ is a polynomial [Sch06, Proposition 5.2], and therefore $E_{s t}(X)$ is a polynomial as well. We have:

$$
a_{2,2}(X)=a_{2,2}\left(X_{0}\right) h^{0}\left(\mathbb{P}^{1}\right)+h_{s t}^{2,1}\left(X_{0}\right) h^{1,0}\left(\mathbb{P}^{1}\right)+h_{s t}^{1,2}\left(X_{0}\right) h^{0,1}\left(\mathbb{P}^{1}\right)+h_{s t}^{1,1}\left(X_{0}\right) h^{1,1}\left(\mathbb{P}^{1}\right) .
$$

As

$$
a_{2,2}\left(X_{0}\right)=h^{2,2}\left(Y_{0}\right)-h^{1,1}(D(1))=61-2 \cdot 45=-29
$$

and

$$
h_{s t}^{1,1}\left(X_{0}\right)=h^{1,1}\left(Y_{0}\right)-h^{0}(D(1))=61-45=16,
$$

we obtain that

$$
a_{2,2}(X)=-29+16=-13 .
$$

Note that Conjecture A holds for $X$ as

$$
h_{s t}^{2,2}(X)=a_{2,2}(X)+\sum_{a_{j}=1} h^{0}\left(D_{j}\right)=-13+45=32 .
$$

7. Outline of the proof. The proof of Theorem E is contained in Sections 8 - 11. In this section we make reference to what is being proved in each of them. 
7.1. For the rest of the paper we use the following.

Notation. Let $X$ be a fourfold with at most Gorenstein terminal singularities and $C \cup S$ the singular locus. We assume that $C$ has pure dimension 1 and is not empty, and $S$ is the finite set of isolated singularities. Let $f: Y \rightarrow X$ be a log-resolution of singularities, which is an isomorphism on the smooth locus of $X$, and $D=\bigcup D_{i}$ the exceptional set. For $D_{i} \subseteq D$, an irreducible component of $D$, we denote $\left.f\right|_{D_{i}}=g_{i}: D_{i} \rightarrow C$. For a general hyperplane section $H \subseteq X$ we denote $H^{\prime}=f^{-1}(H)$. Note that $\left.f\right|_{H^{\prime}}=f^{\prime}: H^{\prime} \rightarrow H$ is a log-resolution.

7.2. The first step of the proof is to get a convenient simplification of

$$
h_{s t}^{2,2}(X)=h^{2,2}(Y)-h^{1,1}(D(1))+h^{0}(D(2))+\sum_{a_{j}=1} h^{0}\left(D_{j}\right) .
$$

Applying Theorem 2.6 we get expressions (8.2) and (8.4), which are direct sum decompositions of $H^{4}(Y)$ and $H^{2}(D(1))$. They include the information of a stratification of $X$ and several local systems. We set the notation for these objects in Section 8.

In Section 9 we either give explicit computations of the dimensions of pieces of these expressions, or results showing that the dimensions of some of these in (8.2) and (8.4) coincide.

Finally, in Section 10 a description of the subspaces $H^{2,2}(Y)$ and $H^{1,1}(D(1))$ is given.

All of the above is put together in Section 11. Using the previous results we obtain a simplification of $h_{s t}^{2,2}(X)$, and we show $h_{s t}^{2,2}(X)-h_{s t}^{1,1}(H) \geq 0$. As the second term is nonnegative by Corollary C, we obtain the result.

8. Set up. Applying Theorem 2.6 to the maps $f$ and $g_{i}$ gives the following data: a stratification of the maps and several local systems. It is convenient to set a clear notation for these objects.

8.1. The stratification of $f$ includes one of $X$ we describe as follows:

Notation. We have

$$
X=X_{\text {reg }} \amalg U \amalg S \amalg S^{\prime} \amalg S^{\prime \prime},
$$

such that:

(a) The space $X_{\text {reg }}=X \backslash C \cup S$ is the open subvariety consisting of the smooth locus.

(b) $U$ is an open set of $C$ contained in the smooth locus of $C$.

(c) $S^{\prime}=\left\{z_{1}, \ldots, z_{r}\right\}$ and $S^{\prime \prime}=\left\{d_{1}, \ldots, d_{s}\right\}$ are a finite set of points in $C$ such that $U \amalg S^{\prime} \amalg S^{\prime \prime}=$ $C$, and $S=\left\{s_{1}, \ldots, s_{t}\right\}$ are the isolated singularities of $X$.

They satisfy the following conditions:

(d) If $C=\bigcup C_{i}$ is reducible, $U=\amalg U_{i}$ where $U_{i} \subseteq C_{i}$ is an open subset contained in the smooth locus of $C_{i}$.

(e) For $d_{i} \in S^{\prime \prime}, f^{-1}\left(d_{i}\right)$ is 3-dimensional.

(f) We have $D=D^{\prime} \cup D^{\prime \prime} \cup D^{\prime \prime \prime}$ and:

(i) for $D_{i}$ a component of $D^{\prime}, f\left(D_{i}\right)=C_{j}$ for some $j$,

(ii) for $D_{j} \subseteq D^{\prime \prime}, f\left(D_{j}\right) \in S^{\prime \prime}$, 
(iii) $D^{\prime \prime \prime}$ is the disjoint union of the fibers of the isolated singularities.

We can assume that for a component $B \subseteq D_{i} \cap D_{j}$, if $f(B)=z \in C$, then $z \in S^{\prime \prime}$ (which implies $D_{i} \subseteq D^{\prime \prime}$ or $\left.D_{j} \subseteq D^{\prime \prime}\right)$. Indeed, we can blow up the connected components of the double intersections of the irreducible components of $D^{\prime}$ that contract to a point. An analogous argument works for triple intersections as well.

We denote $j: U \hookrightarrow C$ the inclusion map. Let $\nu: \tilde{C}=\amalg \tilde{C}_{i} \rightarrow C$ be the normalization and $k: U \rightarrow \tilde{C}$, so that $j=\nu \circ k$. We have a factorization of the map $g_{i}: D_{i} \rightarrow C$, as a composition of $g_{i}^{\prime}: D_{i} \rightarrow \tilde{C}$ and $\nu$, for $D_{i} \subseteq D^{\prime}$. Note that the normalization $\nu$ is an isomorphism on $U$.

8.2. The only intersection complex supported on $X$ in the decomposition of $\mathbf{R} f_{*} \mathbb{C}_{Y}[4]$ is $I C_{X}$, as the map $f$ is birational (cf. [dCM05, Proof of Theorem 2.2.3 d)]). The other intersection complexes are supported on the strata of dimension 1 or 0 , and for these dimensions the intersection complex of a local system has an easy description.

Remark 8.1. For a local system $L$ on $U, I C_{C}(L) \cong j_{*} L[1]$. If $C$ is not smooth, the following isomorphisms are useful:

$$
I C_{C}(L) \cong \nu_{*} I C_{\tilde{C}}(L) \cong \nu_{*} k_{*} L[1] .
$$

For a local system supported on a zero-dimensional space, the intersection complex is the local system itself.

With these, if we apply Theorem 2.6 to $f$, we get the following descriptions:

$$
{ }^{\mathfrak{p}} \mathcal{H}^{k}\left(\mathbf{R} f_{*} \mathbb{C}_{Y}[4]\right)[-k] \cong j_{*} L_{U, k}[1-k] \oplus L_{S, k}[-k] \oplus L_{S^{\prime}, k}[-k] \oplus L_{S^{\prime \prime}, k}[-k]
$$

for $k=-2,-1,1,2$ and

$$
{ }^{\mathfrak{p}} \mathcal{H}^{0}\left(\mathbf{R} f_{*} \mathbb{C}_{Y}[4]\right)[0] \cong I C_{X} \oplus j_{*} L_{U, 0}[1] \oplus L_{S, 0}[0] \oplus L_{S^{\prime}, 0}[0] \oplus L_{S^{\prime \prime}, 0}[0]
$$

where the first subindex corresponds to the stratum in which each local system is defined.

The cohomology $H^{4}(Y)$ is isomorphic to $\mathbb{H}^{0}\left(X, \mathbf{R} f_{*} \mathbb{C}_{Y}[4]\right)$. By applying $\mathbb{H}^{0}$ to the expressions above, we get the following direct sum decomposition:

$$
\begin{aligned}
& H^{0}\left(C, j_{*} L_{U, 1}\right) \\
& H^{4}(Y) \cong \oplus I H^{4}(X) \oplus H^{1}\left(C, j_{*} L_{U, 0}\right) \oplus H^{0}\left(S \amalg S^{\prime} \amalg S^{\prime \prime}, L_{S, 0} \oplus L_{S^{\prime}, 0} \oplus L_{S^{\prime \prime}, 0}\right) \\
& \oplus H^{2}\left(C, j_{*} L_{U,-1}\right)
\end{aligned}
$$

where each line of the sum corresponds to $\mathbb{H}^{0}$ of a perverse cohomology of $\mathbf{R} f_{*} \mathbb{C}_{Y}$ [4].

8.3. Let $D_{i} \subseteq D^{\prime}$ be an irreducible component. The map $g_{i}^{\prime}$ goes form a smooth variety of dimension 3 to a smooth curve. The application of the Decomposition Theorem to these kinds of maps is discussed in [dCM05, Example 2.5]:

Example 8.3. We can assume this map is smooth over $U \subseteq \tilde{C}$. The computation says:

$$
{ }^{\mathfrak{p}} \mathcal{H}^{m}\left(\mathbf{R} g_{i *}^{\prime} \mathbb{C}_{D_{i}}[3]\right) \cong k_{*} R^{2+m} g_{i *}^{\prime} \mathbb{C}_{U_{i}^{\prime}}[1] \oplus K_{i}^{m}
$$


where $U_{i}^{\prime}=g_{i}^{\prime-1}(U)$. Note that by taking the cohomology of the complexes we get

$$
R^{m+3} g_{i *}^{\prime} \mathbb{C}_{D_{i}} \cong k_{*} R^{m+3} g_{i *}^{\prime} \mathbb{C}_{U_{i}^{\prime}} \oplus K_{i}^{m},
$$

which implies that if we take the stalk at $z \in g_{i}^{\prime}\left(D_{i}\right) \backslash U$ we have the following isomorphism:

$$
H^{m+3}\left(g_{i}^{\prime-1}(z)\right) \cong H^{m+3}\left(g_{i}^{\prime-1}(x)\right)^{\mathbb{Z}} \oplus\left(K_{i}^{m}\right)_{z},
$$

where $\mathbb{Z}$ is the monodromy action around the point $z$ and $x \in g_{i}^{\prime}\left(D_{i}\right) \cap U$ is a nearby point. The above discussion gives a description of the sheaves $K_{i}^{m}$.

Using that $H^{2}\left(D_{i}\right)=\mathbb{H}^{-1}\left(\mathbf{R} g_{i *} \mathbb{C}_{D_{i}}[3]\right)=\mathbb{H}^{-1}\left(\nu_{*} \mathbf{R} g_{i *}^{\prime} \mathbb{C}_{D_{i}}[3]\right)$ and that $g_{i}=\nu \circ g_{i}^{\prime}$, we obtain the following direct sum decomposition:

$$
H^{2}\left(D_{i}\right) \cong H^{2}\left(C, j_{*} g_{i *} \mathbb{C}_{U^{\prime}}\right) \oplus H^{1}\left(C, j_{*} R^{1} g_{i *} \mathbb{C}_{U^{\prime}}\right) \oplus H^{0}\left(S, \nu_{*} K_{i}^{-1}\right) \oplus H^{0}\left(U, R^{2} g_{i *} \mathbb{C}_{U^{\prime}}\right) .
$$

9. Computation of the cohomologies. In this section we discuss the summands in expressions (8.2) and (8.4). We also discuss the much simpler expression (9.11), which we obtain by applying Theorem 2.6 to the map $f^{\prime}$.

9.1. We use the notation of Section 8 .

Lemma 9.1. The space of global sections of $L_{U, 1}$ has the following dimension:

$$
h^{0}\left(C, j_{*} L_{U, 1}\right)=h^{0}\left(U, L_{U, 1}\right)=h^{0}\left(D^{\prime}(1)\right) .
$$

Proof. As $\operatorname{dim} \operatorname{Supp} \mathcal{H}^{0}\left(I C_{X}\right)<0$, we have

$$
\mathcal{H}^{0}\left(\mathbf{R} f_{*} \mathbb{C}_{Y}[4]\right)=R^{4} f_{*} \mathbb{C}_{Y} \cong j_{*} L_{U, 1} \oplus L_{S, 0} \oplus L_{S^{\prime}, 0} \oplus L_{S^{\prime \prime}, 0},
$$

and hence

This means that for $x \in U$,

$$
\left.R^{4} f_{*} \mathbb{C}_{Y}\right|_{U} \cong L_{U, 1}
$$

$$
H^{4}\left(f^{-1}(x)\right) \cong\left(R^{4} f_{*} \mathbb{C}_{Y}\right)_{x} \cong\left(L_{U, 1}\right)_{x} .
$$

Let $g: D \rightarrow C \amalg S$ be the restriction of $f$ to $D$. As $R^{4} f_{*} \mathbb{C}_{Y}$ is supported on $C \amalg S$, this sheaf is isomorphic to $R^{4} g_{*} \mathbb{C}_{D}$. The map $\mathbb{C}_{D} \rightarrow \mathbb{C}_{D(1)}$ induces a quasi-isomorphism

$$
\mathbb{C}_{D} \rightarrow\left[0 \rightarrow \mathbb{C}_{D(1)} \rightarrow \ldots \rightarrow \mathbb{C}_{D(4)} \rightarrow 0\right]=: Q
$$

because $D$ is a space with simple normal crossings (see [CEZGL14, Lemma and Definition 3.2.31]). Using the spectral sequence associated to the trivial filtration of the complex $Q$ we compute $R^{4} g_{*} \mathbb{C}_{D}$. It is defined as:

$$
E_{1}^{p, q}=R^{q} g_{*} \mathbb{C}_{D(p+1)} \Rightarrow R^{p+q} g_{*} Q .
$$

We look at $p+q=4$. Note that for $x \in U,\left(R^{4} g_{*} \mathbb{C}_{D(2)}\right)_{x}=0$, as $\operatorname{dim} f^{-1}(x)=1$. Therefore, the maps in the complex

which corresponds to

$$
0 \rightarrow R^{4} g_{*} \mathbb{C}_{D(1)} \rightarrow R^{4} g_{*} \mathbb{C}_{D(2)}
$$

$$
E_{1}^{-1,4} \rightarrow E_{1}^{0,4} \rightarrow E_{1}^{1,4}
$$


are the zero map on $U$. As $E_{1}^{2,3}=0$ by dimension reasons,

$$
\left.\left.R^{4} g_{*} \mathbb{C}_{D(1)}\right|_{U} \cong E_{\infty}^{0,4}\right|_{U}
$$

The terms $E_{1}^{1,3}, E_{1}^{2,2}$ and $E_{1}^{3,1}$ are all zero on $U$ by a similar reasoning. This means that we obtain the isomorphisms

$$
\left.\left.L_{U, 1} \cong R^{4} g_{*} Q\right|_{U} \cong R^{4} g_{*} \mathbb{C}_{D(1)}\right|_{U}
$$

Suppose first $C$ is smooth and irreducible. Example 8.3, implies that

$$
H^{0}\left(C, j_{*} R^{4} g_{i *} \mathbb{C}_{U^{\prime}}\right)=\mathbb{H}^{-1}\left(C,{ }^{\mathfrak{p}} \mathcal{H}^{2}\left(\mathbf{R} g_{i *} \mathbb{C}_{D_{i}}[3]\right)\right) \cong H_{2}^{4}\left(D_{i}\right),
$$

where the last term is the perverse cohomology group with respect to $g_{i}$ (see Definition 2.10). Using Theorem 2.12(i), we get that

$$
H_{-2}^{0}\left(D_{i}\right) \cong H_{2}^{4}\left(D_{i}\right)
$$

and we also have that:

$$
\begin{array}{r}
H_{-2}^{0}\left(D_{i}\right)=\mathbb{H}^{-1}\left(C,{ }^{\mathfrak{p}} \mathcal{H}^{-2}\left(\mathbf{R} g_{i *} \mathbb{C}_{D_{i}}[3]\right)\right)=H^{0}\left(C, j_{*} g_{i *} \mathbb{C}_{U^{\prime}}\right) \\
=H^{0}\left(U, g_{i *} \mathbb{C}_{U^{\prime}}\right) \cong H^{0}\left(U^{\prime}, \mathbb{C}_{U^{\prime}}\right) .
\end{array}
$$

Hence,

$$
H^{4}\left(U, R^{4} g_{i *} \mathbb{C}_{U^{\prime}}\right) \cong H^{0}\left(C, j_{*} R^{4} g_{i *} \mathbb{C}_{U^{\prime}}\right) \cong H^{0}\left(U^{\prime}, \mathbb{C}_{U^{\prime}}\right)
$$

and

$$
\operatorname{dim} H^{4}\left(U, R^{4} g_{i *} \mathbb{C}_{U^{\prime}}\right)=1
$$

To conclude, since we have

$$
H^{0}\left(U, L_{U, 1}\right) \cong H^{0}\left(U,\left.R^{4} g_{*} \mathbb{C}_{D(1)}\right|_{U}\right)
$$

by (9.2), we obtain that

$$
\operatorname{dim} H^{0}\left(U, L_{U, 1}\right)=\sum \operatorname{dim} H^{0}\left(U,\left.R^{4} g_{*} \mathbb{C}_{D_{i}}\right|_{U}\right)=h^{0}\left(D^{\prime}(1)\right) .
$$

Assume now that $C$ is an arbitrary curve. For every $D_{i} \subseteq D^{\prime}$, the map $g_{i}^{\prime}$ surjects onto a smooth and irreducible curve $\tilde{C}_{j}$. We can use the same analysis for this map, and get the same conclusion.

Lemma 9.3. The following holds:

$$
h^{2}\left(C, j_{*} L_{U,-1}\right)=h^{0}\left(D^{\prime}(1)\right) .
$$

Proof. This is a simple application of Theorem 2.12. We have

$$
H_{1}^{4}(Y)=\mathbb{H}^{-1}\left(X,{ }^{\mathfrak{p}} \mathcal{H}^{1}\left(\mathbf{R} f_{*} \mathbb{C}_{Y}[4]\right)\right) \cong H^{0}\left(U, L_{U, 1}\right),
$$

and using Theorem 2.12(i) we get that

$$
H_{-1}^{2}(Y) \cong H_{1}^{4}(Y)
$$

Theorem 2.12(ii) implies that

$$
H_{-1}^{2}(Y) \cong H_{-1}^{4}(Y)
$$

and finally note that

$$
H_{-1}^{4}(Y)=\mathbb{H}^{1}\left(X,{ }^{\mathfrak{p}} \mathcal{H}^{-1}\left(\mathbf{R} f_{*} \mathbb{C}_{Y}[4]\right)\right) \cong H^{2}\left(C, j_{*} L_{U,-1}\right)
$$


The result follows from Lemma 9.1, as

$$
\operatorname{dim} H_{1}^{4}(Y)=h^{0}\left(U, L_{U, 1}\right)=h^{0}\left(D^{\prime}(1)\right)
$$

Remark 9.4. In the proof we have shown that there is a chain of isomorphisms of type $(1,1)$ between these pure Hodge structures. This is used in Section 10 when we discuss the subspace $H^{2,2}(Y)$.

Lemma 9.5. The following terms in (8.2) and (8.4) have the same dimension:

$$
h^{1}\left(C, j_{*} L_{U, 0}\right)=\sum h^{1}\left(C, j_{*} R^{1} g_{i *} \mathbb{C}_{U^{\prime}}\right)
$$

Proof. Using that $R^{3} f_{*} \mathbb{C}_{Y}=\mathcal{H}^{-1}\left(\mathbf{R} f_{*} \mathbb{C}_{Y}[4]\right)$, and (8.2), we have

$$
R^{3} f_{*} \mathbb{C}_{Y} \cong \mathcal{H}^{-1}\left(I C_{X}\right) \oplus j_{*} L_{U, 0} \oplus L_{S,-1} \oplus L_{S^{\prime},-1} .
$$

As $\operatorname{dim} \operatorname{Supp} \mathcal{H}^{-1}\left(I C_{X}\right)<1$, it is supported on a finite set of points, and we can assume they are not in $U$. We use again the quasi-isomorphism

$$
\mathbb{C}_{D} \rightarrow\left[0 \rightarrow \mathbb{C}_{D}(1) \rightarrow \ldots \rightarrow \mathbb{C}_{D(4)} \rightarrow 0\right]=: Q,
$$

and the spectral sequence

$$
E_{1}^{p, q}=R^{q} g_{*} \mathbb{C}_{D(p+1)} \Rightarrow R^{p+q} g_{*} Q
$$

(see the proof of Lemma 9.1). We consider the terms with $p+q=3$. For $x \in U,\left(R^{3} g_{*} \mathbb{C}_{D(2)}\right)_{x}=0$ by dimension reasons. The maps of the complex

$$
0 \rightarrow R^{3} g_{*} \mathbb{C}_{D(1)} \rightarrow R^{3} g_{*} \mathbb{C}_{D(2)},
$$

which corresponds to

$$
E_{1}^{-1,3} \rightarrow E_{1}^{0,3} \rightarrow E_{1}^{1,3}
$$

are the zero map on $U$. The term $\left.E_{1}^{2,2}\right|_{U}=0$ as well. We get

$$
\left.\left.R^{3} g_{*} \mathbb{C}_{D(1)}\right|_{U} \cong E_{\infty}^{0,3}\right|_{U}
$$

Analogous arguments show $E_{1}^{2,1}$ and $E_{1}^{3,0}$ are all zero on $U$.

Consider the maps

which correspond to

$$
R^{2} g_{*} \mathbb{C}_{D(1)} \rightarrow R^{2} g_{*} \mathbb{C}_{D(2)} \rightarrow R^{2} g_{*} \mathbb{C}_{D(3)},
$$

$$
E_{1}^{0,2} \rightarrow E_{1}^{1,2} \rightarrow E_{1}^{2,2}
$$

For $x \in U$, the map on stalks corresponds to the alternate sum of restriction maps

$$
\bigoplus H^{2}\left(g_{i}^{-1}(x)\right) \stackrel{\alpha}{\rightarrow} \bigoplus H^{2}\left(g_{i}^{-1}(x) \cap g_{j}^{-1}(x)\right) .
$$

The space $f^{-1}(x)$ is a surface with simple normal crossings, the fibers $g_{i}^{-1}(x)$ are smooth, and are the components of $f^{-1}(x)$. Therefore,

$$
\operatorname{ker} \alpha \cong \operatorname{Gr}_{2}^{W}\left(H^{2}\left(f^{-1}(x)\right)\right)
$$


and

$$
\text { coker } \alpha \cong \operatorname{Gr}_{2}^{W}\left(H^{3}\left(f^{-1}(x)\right)\right)
$$

(see (2.5)). The surface $f^{-1}(x)$ shows up in a resolution of a threefold with isolated singularities, and hence

$$
\text { coker } \alpha=0
$$

[Ste83, Corollary 1.12]. This means that $\left.E_{2}^{1,2}\right|_{U}=0$.

We obtain that

$$
\left.R^{3} g_{*} \mathbb{C}_{D(1)}\right|_{U} \cong L_{U, 0}
$$

In particular,

$$
\operatorname{dim} H^{1}\left(C, j_{*} R^{3} g_{*} \mathbb{C}_{U^{\prime}}\right)=\operatorname{dim} H^{1}\left(C, j_{*} L_{U, 0}\right) .
$$

Suppose $C$ is smooth and irreducible. We have:

$$
H_{1}^{4}\left(D_{i}\right) \cong H^{1}\left(C, j_{*} R^{3} g_{*} \mathbb{C}_{U^{\prime}}\right) \oplus H^{0}\left(S^{\prime}, K_{i}^{1}\right)
$$

and

$$
H_{-1}^{2}\left(D_{i}\right) \cong H^{1}\left(C, j_{*} R^{1} g_{*} \mathbb{C}_{U^{\prime}}\right) \oplus H^{0}\left(S^{\prime}, K_{i}^{-1}\right)
$$

and they are isomorphic by Theorem 2.12(i). We also have

$$
H^{0}\left(S^{\prime}, K_{i}^{1}\right) \cong H^{0}\left(S^{\prime}, K_{i}^{-1}\right)
$$

as they are dual to each other (see for instance [dCM07, Section 4.4]). Therefore, we get

$$
\operatorname{dim} H^{1}\left(C, j_{*} R^{3} g_{i *} \mathbb{C}_{U^{\prime}}\right)=\operatorname{dim} H^{1}\left(C, j_{*} R^{1} g_{i *} \mathbb{C}_{U^{\prime}}\right)
$$

The result follows from (9.6) and (9.7).

With no restriction on $C$, we use the map $g_{i}^{\prime}: D_{i} \rightarrow \tilde{C}$. By the previous analysis

$$
\operatorname{dim} H^{1}\left(\tilde{C}, k_{*} R^{3} g_{i *}^{\prime} \mathbb{C}_{U^{\prime}}\right)=\operatorname{dim} H^{1}\left(\tilde{C}, k_{*} R^{1} g_{i *}^{\prime} \mathbb{C}_{U^{\prime}}\right) .
$$

Since

$$
\operatorname{dim} H^{1}\left(\tilde{C}, k_{*} R^{3} g_{i *}^{\prime} \mathbb{C}_{U^{\prime}}\right)=\operatorname{dim} H^{1}\left(C, j_{*} R^{3} g_{i *}^{\prime} \mathbb{C}_{U^{\prime}}\right)
$$

and

$$
\operatorname{dim} H^{1}\left(\tilde{C}, k_{*} R^{1} g_{i *}^{\prime} \mathbb{C}_{U^{\prime}}\right)=\operatorname{dim} H^{1}\left(C, j_{*} R^{1} g_{i *}^{\prime} \mathbb{C}_{U^{\prime}}\right),
$$

because $\nu$ is finite, the result follows.

This concludes the discussion of terms which include intersection complexes supported on the 1-dimensional strata. We examine next those with local systems supported on the 0-dimensional strata.

Lemma 9.8. The following dimensions of terms in (8.2) and (8.4) coincide:

$$
h^{0}\left(S^{\prime}, L_{S^{\prime}, 0}\right)=\sum h^{0}\left(S^{\prime}, \nu_{*} K_{i}^{-1}\right) .
$$


Proof. Let $z \in S^{\prime}$. As explained in Lemma 9.1,

$$
\left(R^{4} f_{*} \mathbb{C}_{Y}\right)_{z} \cong\left(j_{*} L_{U, 1}\right)_{z} \oplus L_{z, 0}
$$

and

$$
\left(R^{4} g_{i *} \mathbb{C}_{D_{i}}\right)_{z} \cong\left(j_{*} R^{4} g_{i *} \mathbb{C}_{U^{\prime}}\right)_{z} \oplus\left(\nu_{*} K_{i}^{1}\right)_{z} .
$$

Using the quasi-isomorphism

$$
\mathbb{C}_{D} \rightarrow Q
$$

and the spectral sequence

$$
E_{1}^{p, q}=\left(R^{q} g_{*} \mathbb{C}_{D(p+1)}\right)_{z} \Rightarrow\left(R^{p+q} g_{*} Q\right)_{z},
$$

we compute $\left(R^{4} g_{*} Q\right)_{z}$ (see proof of Lemma 9.1).

We look at the terms with $p+q=4$. The maps

$$
0 \rightarrow\left(R^{4} g_{*} \mathbb{C}_{D(1)}\right)_{z} \rightarrow\left(R^{4} g_{*} \mathbb{C}_{D(2)}\right)_{z}
$$

corresponding to

$$
E_{1}^{-1,4} \rightarrow E_{1}^{0,4} \rightarrow E_{1}^{1,4}
$$

are the zero map. Indeed, if $\left(R^{4} g_{*} \mathbb{C}_{D(2)}\right)_{z}=H^{4}\left(g_{i}^{-1}(z) \cap g_{j}^{-1}(z)\right) \neq 0$, then a component of $D_{i} \cap D_{j}$ is contracted to a point. By assumption this is not possible, because $z \notin S^{\prime \prime}$. As $E_{1}^{2,3}=0$ by the same reason, we see that

$$
E_{1}^{0,4}=E_{\infty}^{0,4}
$$

In the same way, $E_{1}^{1,3}, E_{1}^{2,2}$ and $E_{1}^{3,1}$ are all zero. Hence

$$
\left(R^{4} g_{*} \mathbb{C}_{D(1)}\right)_{z} \cong\left(R^{4} g_{*} Q\right)_{z} \cong\left(R^{4} f_{*} \mathbb{C}_{Y}\right)_{z} .
$$

By Lemma 9.1 we have

which implies

$$
L_{U, 1} \cong \bigoplus R^{4} g_{i *} \mathbb{C}_{U^{\prime}}
$$

$$
\operatorname{dim} H^{0}\left(S^{\prime}, L_{S^{\prime}, 0}\right)=\sum \operatorname{dim} H^{0}\left(S^{\prime}, \nu_{*} K_{i}^{1}\right)
$$

by adding the dimension of the stalks of every $z \in S^{\prime}$. Using

$$
H^{0}\left(S^{\prime}, \nu_{*} K_{i}^{-1}\right) \cong H^{0}\left(S^{\prime}, \nu_{*} K_{i}^{1}\right)
$$

the result follows.

Lemma 9.9. For $d \in S^{\prime \prime}$

$$
\begin{aligned}
\operatorname{dim} H^{2,2}\left(H^{0}\left(L_{d, 0}\right)\right) & =h^{1,1}\left(f^{-1}(d)(1)\right)-h^{0}\left(f^{-1}(d)(2)\right) \\
& -\sum_{\substack{x_{j} \text { near } \\
d_{j} \in \nu^{-1}(d)}}\left(\operatorname{dim} H^{2,2}\left(H^{4}\left(f^{-1}\left(x_{j}\right)\right)^{\mathbb{Z}}\right)\right)
\end{aligned}
$$

where $f^{-1}(d)(1)$ and $f^{-1}(d)(2)$ is the usual notation for a simple normal crossings divisor (see Section 1.3). 
Proof. From (8.2) we get

$$
H^{4}\left(f^{-1}(d)\right) \cong\left(j_{*} L_{U, 1}\right)_{d} \oplus L_{d, 0} .
$$

If $d \notin g_{i}\left(D_{i}\right)$, then $\left(j_{*} R^{4} g_{i *} \mathbb{C}_{U^{\prime}}\right)_{d}=0$. Otherwise using the isomorphism (9.2) we obtain,

$$
\begin{aligned}
\left(j_{*} L_{U, 1}\right)_{d}=\left(\nu_{*} k_{*} L_{U, 1}\right)_{d} & =\bigoplus_{d_{j} \in \nu^{-1}(d)}\left(k_{*} L_{U, 1}\right)_{d_{j}} \\
& \cong \bigoplus_{d_{j} \in \nu^{-1}(d)} H^{4}\left(f^{-1}\left(x_{j}\right)\right)^{\mathbb{Z}}
\end{aligned}
$$

where $x_{j} \in g_{i}\left(D_{i}\right) \cap U$ is near $d_{j}$, and $\mathbb{Z}$ is the monodromy around the point $d_{j}$. The result follows from the computation of the $H^{2,2}$ piece of the cohomology of a simple normal crossings divisor (see Section 2.3).

Lemma 9.10. For the local system supported on the isolated singularities of $X$,

$$
h^{2,2}\left(H^{0}\left(S, L_{S, 0}\right)\right)=h^{1,1}\left(D^{\prime \prime \prime}(1)\right)-h^{0}\left(D^{\prime \prime \prime}(2)\right) .
$$

Proof. Let $s \in S$. In this case we have that

$$
H^{4}\left(f^{-1}(s)\right) \cong\left(R^{4} f_{*} \mathbb{C}_{Y}\right)_{s} \cong L_{s, 0}
$$

We know that $H^{0}\left(s, L_{s, 0}\right)$ is a pure Hodge substructure of $H^{4}(Y)$, and $f^{-1}(s)$ must be a simple normal crossings divisor. Therefore,

$$
\begin{aligned}
h^{2,2}\left(H^{0}\left(s, L_{s, 0}\right)\right)=h^{2,2}\left(f^{-1}(s)\right) & =h^{2,2}\left(f^{-1}(s)(1)\right)-h^{2,2}\left(f^{-1}(s)(2)\right) \\
& =h^{1,1}\left(f^{-1}(s)(1)\right)-h^{0}\left(f^{-1}(s)(2)\right)
\end{aligned}
$$

(see Section 2.3).

9.2. We discuss the application of Theorem 2.6 to the map $f^{\prime}$. Let $T=U \cap H=\left\{x_{j, l}\right\}$, where $x_{j, l} \in U_{j} \subseteq C_{j}$. As it is a log-resolution of singularities of a threefold with isolated singularities, we have:

$$
\begin{aligned}
&{ }^{\mathfrak{p}} \mathcal{H}^{1}\left(\mathbf{R} f_{*}^{\prime} \mathbb{C}_{H^{\prime}}[3]\right)[-1] \cong \bigoplus H^{4}\left(f^{-1}\left(x_{j, l}\right)\right)_{x_{j, l}}[-1] \\
&{ }^{\mathfrak{p}} \mathcal{H}^{0}\left(\mathbf{R} f_{*}^{\prime} \mathbb{C}_{H^{\prime}}[3]\right)[0] \cong I C_{H} \oplus \bigoplus H^{3}\left(f^{-1}\left(x_{j, l}\right)\right)_{x_{j, l}}[0] \\
&{ }^{\mathfrak{p}} \mathcal{H}^{-1}\left(\mathbf{R} f_{*}^{\prime} \mathbb{C}_{H^{\prime}}[3]\right)[1] \cong \bigoplus H_{4}\left(f^{-1}\left(x_{j, l}\right)\right)_{x_{j, l}}[1] .
\end{aligned}
$$

by the computation in [dCM07, Remark 4.4.3].

We obtain that

$$
H^{2}\left(H^{\prime}\right) \cong \mathbb{H}^{-1}\left(H, \mathbf{R} f_{*}^{\prime} \mathbb{C}_{H^{\prime}}[3]\right) \cong I H^{2}(H) \oplus \bigoplus H_{4}\left(f^{-1}\left(x_{j, l}\right)\right)
$$

We also have that the inclusion $H_{4}\left(f^{-1}\left(x_{j, l}\right)\right) \subseteq H^{2}\left(H^{\prime}\right)$ is given by the composition

$$
H_{4}\left(f^{-1}\left(x_{j, l}\right)\right) \hookrightarrow H_{4}\left(H^{\prime}\right) \cong H^{2}\left(H^{\prime}\right) .
$$


The space $H_{4}\left(f^{-1}\left(x_{j, l}\right)\right)$ is generated by the classes of the irreducible components of $f^{-1}\left(x_{j, l}\right)$, and its image is contained in $H^{1,1}\left(H^{\prime}\right) \subseteq H^{2}\left(H^{\prime}\right)$.

9.3. We discuss next the terms in expression (8.4) which were not part of the results of Section 9.1 .

Lemma 9.12. The spaces $H^{2}\left(C, j_{*} g_{i *} \mathbb{C}_{U^{\prime}}\right)$ are 1-dimensional.

Proof. We have

$$
H_{-2}^{2}\left(D_{i}\right)=\mathbb{H}^{1}\left(C,{ }^{\mathfrak{p}} \mathcal{H}^{-2}\left(\mathbf{R} g_{i *} \mathbb{C}_{D_{i}}[3]\right)\right) \cong H^{2}\left(C, j_{*} g_{i *} \mathbb{C}_{U^{\prime}}\right),
$$

and Theorem 2.12(ii) implies

$$
H_{-2}^{0}\left(D_{i}\right) \cong H_{-2}^{2}\left(D_{i}\right) .
$$

The result follows, as $\operatorname{dim} H_{-2}^{0}\left(D_{i}\right)=1$ (see proof of Lemma 9.1).

Consider the Stein factorization

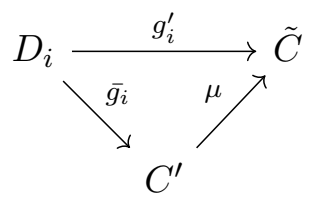

where $\mu$ is a finite map and $\bar{g}_{i}$ has connected fibers. The map $\bar{g}_{i}$ is smooth in the preimage of an open set of $C^{\prime}$, and $\mu$ has no branch points in an open set of $C^{\prime}$. We can assume $U$ is contained in the image of these open sets. Let $x \in U$ and $\mu^{-1}(x)=\left\{y_{1}, \ldots, y_{l_{i}}\right\}$.

Lemma 9.13. With the notation above

$$
H^{0}\left(U, R^{2} g_{i *} \mathbb{C}_{U^{\prime}}\right) \cong H^{0}\left(U, R^{2} g_{i *}^{\prime} \mathbb{C}_{U^{\prime}}\right) \cong \operatorname{im}\left\{H^{2}\left(D_{i}\right) \rightarrow H^{2}\left(\bar{g}_{i}{ }^{-1}\left(y_{j}\right)\right)\right\}
$$

for any $j$.

Proof. As the surfaces $\bar{g}_{i}^{-1}\left(y_{j}\right)$ are fibers of the smooth map $\left.\bar{g}_{i}\right|_{U^{\prime}}$, they are all diffeomorphic. The map $\mu$ is finite, so $\mu_{*} R^{2} \bar{g}_{i *} \mathbb{C}_{U^{\prime}} \cong R^{2} g_{i *}^{\prime} \mathbb{C}_{U^{\prime}}$. The Leray spectral sequence implies

$$
H^{0}\left(U, R^{2} g_{i *}^{\prime} \mathbb{C}_{U^{\prime}}\right) \cong H^{0}\left(\mu^{-1}(U), R^{2} \overline{g_{i *}} \mathbb{C}_{U^{\prime}}\right)
$$

The second isomorphism is a consequence of applying Theorem 2.11 to $\bar{g}_{i}$, and the isomorphism above. The first isomorphism is a consequence of $\nu$ being a finite map.

10. Hodge structures. In this section we describe the subspaces $H^{2,2}(Y)$ and $H^{1,1}\left(D_{i}\right)$.

10.1. We describe first the $H^{2,2}$ pieces of the summands in (8.2).

Lemma 10.1. The following holds:

$$
H^{0}\left(U, L_{U, 1}\right) \subseteq H^{2,2}(Y) \subseteq H^{4}(Y)
$$

and

$$
H^{2}\left(C, j_{*} L_{U,-1}\right) \subseteq H^{2,2}(Y) \subseteq H^{4}(Y)
$$


Proof. Recall that

$$
H_{-1}^{2}(Y) \cong H^{0}\left(U, L_{U,-1}\right)
$$

and

$$
H_{-1}^{2}\left(H^{\prime}\right) \cong \bigoplus_{i=1}^{k} H_{4}\left(f^{-1}\left(x_{i}\right)\right)
$$

(see Definition 2.10). The map $H^{2}(Y) \stackrel{v^{*}}{\rightarrow} H^{2}\left(H^{\prime}\right)$ respects the perverse filtration by Lemma 2.7. Proposition 2.9 implies that

$$
\mathbb{H}^{-1}\left(X,{ }^{\mathfrak{p}} \mathcal{H}^{-1}\left(\mathbf{R} f_{*} \mathbb{C}_{Y}[4]\right)\right) \rightarrow \mathbb{H}^{-1}\left(H, u^{* \mathfrak{p}} \mathcal{H}^{-1}\left(\mathbf{R} f_{*} \mathbb{C}_{Y}[4]\right)\right)
$$

is an injection. By Lemma 2.7

$$
u^{* \mathfrak{p}} \mathcal{H}^{-1}\left(\mathbf{R} f_{*} \mathbb{C}_{Y}[4]\right) \cong{ }^{\mathfrak{p}} \mathcal{H}^{-1}\left(\mathbf{R} f_{*}^{\prime} \mathbb{C}_{H^{\prime}}[3]\right)[1]
$$

and so

$$
\mathbb{H}^{-1}\left(H, u^{* \mathfrak{p}} \mathcal{H}^{-1}\left(\mathbf{R} f_{*} \mathbb{C}_{Y}[4]\right)\right) \cong \mathbb{H}^{0}\left(H,{ }^{\mathfrak{p}} \mathcal{H}^{-1}\left(\mathbf{R} f_{*}^{\prime} \mathbb{C}_{H^{\prime}}[3]\right)\right)=H_{-1}^{2}\left(H^{\prime}\right) .
$$

The conclusion is that

$$
H_{-1}^{2}(Y) \rightarrow H_{-1}^{2}\left(H^{\prime}\right)
$$

is an inclusion. As the restriction map is one of Hodge structures, and

$$
H_{-1}^{2}\left(H^{\prime}\right) \subseteq H^{1,1}\left(H^{\prime}\right)
$$

as discussed in Section 9.2, we obtain:

$$
H_{-1}^{2}(Y) \subseteq H^{1,1}(Y)
$$

As explained in Remark 9.4,

$$
H_{-1}^{2}(Y) \cong H_{1}^{4}(Y)
$$

via a $(1,1)$-map, hence

$$
H_{1}^{4}(Y) \subseteq H^{2,2}(Y)
$$

Analogously, we get

$$
H_{-1}^{4}(Y) \subseteq H^{2,2}(Y)
$$

10.2. Finally we describe the $H^{1,1}$ pieces of the summands of (8.4).

Lemma 10.2. The space $H^{2}\left(C, j_{*} g_{i *} \mathbb{C}_{U^{\prime}}\right)$ is contained in $H^{1,1}\left(D_{i}\right)$.

Proof. In the proof of Lemma 9.12 it was shown that

$$
H_{-2}^{0}\left(D_{i}\right) \cong H_{-2}^{2}\left(D_{i}\right)
$$

via a $(1,1)$ map. As $H^{0}\left(D_{i}\right)=H^{0,0}\left(D_{i}\right)$, the result follows.

11. Proof. In this section we prove Theorem E. 
11.1. The theorem is proved assuming condition $(*)$. Recall the definition:

If $D \subseteq Y$ is the exceptional set of $f$, with irreducible components $D=\bigcup D_{i}$, $\left.f\right|_{D_{i}}$ has connected fibers, and for any irreducible component $B_{i j} \subseteq D_{i} \cap D_{j}$, $\left.f\right|_{B_{i j}}$ has connected fibers.

Proof of Theorem E. We show first that

$$
h_{s t}^{2,2}(X) \geq h_{s t}^{1,1}(H)
$$

for a general hyperplane $H \subseteq X$. This inequality is equivalent to:

$$
h^{2,2}(Y)-h^{1,1}(D(1))+h^{0}(D(2))+\sum_{a_{i}=1} h^{0}\left(D_{i}\right)-h^{1,1}\left(H^{\prime}\right)+h^{0}(E(1)) \geq 0
$$

where $E=D \cap H^{\prime}$.

Consider the first part of the sum:

$$
h^{2,2}(Y)-h^{1,1}(D(1)) .
$$

Combining the Lemmas in Sections 9 and 10 we get

$$
\begin{aligned}
& h^{2,2}(Y)-h^{1,1}(D(1))=\left[2 h^{0}\left(D^{\prime}(1)\right)+\operatorname{dim} H^{2,2}\left(I H^{4}(X)\right)-h^{0}\left(D^{\prime \prime}(2)\right)-h^{0}\left(D^{\prime \prime \prime}(2)\right)\right. \\
- & \left.\sum_{d \in S^{\prime \prime}} \sum_{\substack{x_{j} \\
d_{j} \in \nu^{-1}(d)}}\left(\operatorname{dim} H^{2,2}\left(H^{4}\left(f^{-1}\left(x_{j}\right)\right)^{\mathbb{Z}}\right)\right)\right]-\left[h^{0}\left(D^{\prime}(1)\right)+\sum h^{1,1}\left(H^{0}\left(U, R^{2} g_{i *} \mathbb{C}_{U^{\prime}}\right)\right)\right]
\end{aligned}
$$

after canceling the terms of Lemma 9.5 and Lemma 9.8.

Note that

$$
h^{0}\left(f^{-1}(x)(1)\right) \geq \operatorname{dim} H^{2,2}\left(H^{4}\left(f^{-1}(x)\right)^{\mathbb{Z}}\right) .
$$

Using this, together with the discussion of Section 9.2, we obtain the following inequality:

$$
\begin{aligned}
h_{s t}^{2,2}(X)-h_{s t}^{1,1}(H) & \geq h^{0}\left(D^{\prime}(1)\right)+\operatorname{dim} H^{2,2}\left(I H^{4}(X)\right)-\operatorname{dim} H^{1,1}\left(I H^{2}(H)\right) \\
& -h^{0}\left(D^{\prime \prime}(2)\right)-h^{0}\left(D^{\prime \prime \prime}(2)\right)+h^{0}(D(2))+\sum_{a_{i}=1} h^{0}\left(D_{i}\right) \\
& -\sum_{d \in S^{\prime \prime}} \sum_{\substack{x_{j} \text { near } \\
d_{j} \in \nu^{-1}(d)}} h^{0}\left(f^{-1}\left(x_{j}\right)(1)\right)-\sum h^{1,1}\left(H^{0}\left(U, R^{2} g_{i *} \mathbb{C}_{U^{\prime}}\right)\right) .
\end{aligned}
$$

Applying Theorem 2.13 we get

$$
I H^{2}(X) \cong I H^{2}(H)
$$

and Theorem 2.14 implies there is an injection

$$
I H^{2}(X) \hookrightarrow I H^{4}(X) .
$$

These maps respect the Hodge structures of the intersection cohomologies, and therefore

$$
\operatorname{dim} H^{2,2}\left(I H^{4}(X)\right)-\operatorname{dim} H^{1,1}\left(I H^{2}(H)\right) \geq 0 .
$$


Next, by the definitions of $D=D^{\prime} \cup D^{\prime \prime} \cup D^{\prime \prime \prime}$ we have

$$
h^{0}(D(2))=h^{0}\left(D^{\prime}(2)\right)+h^{0}\left(D^{\prime \prime}(2)\right)+\sum_{\substack{D_{i} \subseteq D^{\prime} \\ D_{j} \subseteq D^{\prime \prime}}} h^{0}\left(D_{i} \cap D_{j}\right)+h^{0}\left(D^{\prime \prime \prime}(2)\right) .
$$

As the components of $D^{\prime \prime \prime}$ do not intersect $D^{\prime}$ nor $D^{\prime \prime}$, because they are the fibers of the isolated singularities, these terms cancel out in the right hand side of the inequality above. On the other hand, as we are assuming condition $(*)$, the general fiber of $\left.f\right|_{D_{i}}$ is connected, hence for each component of $D^{\prime}$ corresponds one component of $f^{-1}(x)$. Also, every $D_{i} \subseteq D^{\prime}$ must intersect a component of $f^{-1}(d)$, for all $d \in S^{\prime \prime}$. This means that

$$
\sum_{\substack{D_{i} \subseteq D^{\prime} \\ D_{j} \subseteq D^{\prime \prime}}} h^{0}\left(D_{i} \cap D_{j}\right) \geq \sum_{d \in S^{\prime \prime}} \sum_{\substack{x_{j} \text { near } \\ d_{j} \in \nu^{-1}(d)}} h^{0}\left(f^{-1}\left(x_{j}\right)(1)\right) .
$$

Note that if $\nu^{-1}(d) \cap \tilde{C}_{j}$ has $k$ points, then for every $D_{i}$ such that $g_{i}\left(D_{i}\right)=C_{j}, D_{i} \cap f^{-1}(d)$ has at least $k$ components. Indeed, $g_{i}^{-1}(d)=D_{i} \cap f^{-1}(d)$, and $g_{i}=\nu \circ g_{i}^{\prime}$.

With the discussion above we can simplify the inequality:

$$
\begin{aligned}
h_{s t}^{2,2}(X)-h_{s t}^{1,1}(H) & \geq h^{0}\left(D^{\prime}(1)\right)+h^{0}\left(D^{\prime}(2)\right) \\
& -\sum h^{1,1}\left(H^{0}\left(U, R^{2} g_{i *} \mathbb{C}_{U^{\prime}}\right)\right)+\sum_{a_{i}=1} h^{0}\left(D_{i}\right) .
\end{aligned}
$$

We also have

$$
h^{1,1}\left(g_{i}^{-1}(x)\right) \geq h^{1,1}\left(H^{0}\left(U, R^{2} g_{i *} \mathbb{C}_{U^{\prime}}\right)\right),
$$

that comes from assuming the local system has trivial monodromy, and note that

$$
\sum h^{1,1}\left(g_{i}^{-1}(x)\right)=h^{1,1}\left(f^{-1}(x)(1)\right) .
$$

We obtain

$$
h_{s t}^{2,2}(X)-h_{s t}^{1,1}(H) \geq h^{0}\left(D^{\prime}(1)\right)+h^{0}\left(D^{\prime}(2)\right)-\sum h^{1,1}\left(f^{-1}\left(x_{k}\right)(1)\right)+\sum_{a_{i}=1} h^{0}\left(D_{i}\right)
$$

where for every connected component of $U$, say $U_{k}$, we pick $x_{k} \in U_{k}$.

Consider the right hand side of the inequality. Recall $T=U \cap H=\left\{x_{j, l}\right\}$ with $x_{j, l} \in U_{j} \subseteq C_{j}$. We want to compare it to a piece of the sum

$$
h_{s t}^{2,2}(H)-h_{s t}^{1,1}(H)=-h^{1,1}(E(1))+h^{0}(E(2))+\sum_{a_{k l i}=1} h^{0}\left(E_{k l i}\right)+h^{0}(E(1)) .
$$

Recall that $f^{-1}\left(x_{k, l}\right)=E_{k l}=E_{k l 1} \cup \cdots \cup E_{k l m}$. If we fix $k$ and vary $l$, the fibers do not intersect, they all have the same number of components, double intersections, and components with discrepancy one. This means that the sum similar to the one right hand side above, but with $E_{k l}$ instead of $E$, is the same for all $l$. 
We fix $l$ from now on. As to each $D_{i} \subseteq D^{\prime}$ such that $g_{i}\left(D_{i}\right)=C_{k}$ corresponds one component of $E_{k l}$, namely $E_{k l i}$, we have

$$
h^{0}\left(D^{\prime}(1)\right)=\sum_{k} h^{0}\left(E_{k l}(1)\right) .
$$

Also, the corresponding discrepancies are the same, hence

$$
\sum_{a_{i}=1} h^{0}\left(D_{i}\right) \geq \sum_{a_{k l i}=1} h^{0}\left(E_{k l i}\right)
$$

as some components of $D^{\prime \prime}$ might have discrepancy 1 . It is also clear that

$$
h^{1,1}\left(f^{-1}\left(x_{k}\right)(1)\right)=h^{1,1}\left(f^{-1}\left(x_{k, l}\right)(1)\right)=h^{1,1}\left(E_{k l}(1)\right) .
$$

Finally, as we are also assuming the general fibers of the maps $\left.g_{i}\right|_{D_{i} \cap D_{j}}$ are connected in each of the components of $D_{i} \cap D_{j}$ in condition (*), we have

$$
h^{0}\left(D^{\prime}(2)\right)=\sum_{k} h^{0}\left(E_{k l}(2)\right)
$$

We obtain:

$$
\begin{aligned}
h_{s t}^{2,2}(H)-h_{s t}^{1,1}(H) & =h^{0}(E(1))+h^{0}(E(2))-h^{1,1}(E(1))+\sum_{a_{k l i}=1} h^{0}\left(E_{k l i}\right) \\
& =\sum_{k, l}\left(h^{0}\left(E_{k l}(1)\right)+h^{0}\left(E_{k l}(2)\right)-h^{1,1}\left(E_{k l}(1)\right)+\sum_{a_{k l i}=1} h^{0}\left(E_{k l i}\right)\right)
\end{aligned}
$$

The conclusion of the discussion above is that

$$
\begin{aligned}
& h^{0}\left(D^{\prime}(1)\right)+h^{0}\left(D^{\prime}(2)\right)-\sum h^{1,1}\left(f^{-1}\left(x_{k}\right)(1)\right)+\sum_{a_{i}=1} h^{0}\left(D_{i}\right) \\
\geq & \sum_{k}\left(h^{0}\left(E_{k l}(1)\right)+h^{0}\left(E_{k l}(2)\right)-h^{1,1}\left(E_{k l}(1)\right)+\sum_{a_{k l i}=1} h^{0}\left(E_{k l i}\right)\right) .
\end{aligned}
$$

The right hand side is a piece of $h_{s t}^{2,2}(H)-h_{s t}^{1,1}(H)$, which consists of picking one fiber per curve, that is, fixing a value of $l$. In Proposition 4.5 it was shown that this number is nonnegative, and therefore

$$
h_{s t}^{2,2}(X)-h_{s t}^{1,1}(H) \geq 0 .
$$

Applying Corollary $\mathrm{C}$ to $H$ we obtain

$$
h_{\text {st }}^{2,2}(X) \geq 0
$$

12. Applications. In this section we discuss classes of fourfolds $X$ for which $h_{s t}^{2,2}(X)$ is nonnegative. 
12.1. We discuss first the example of a product.

Example 12.1. Let $X_{0}$ be a threefold with at most terminal Gorenstein singularities and $C$ a smooth curve. Let $Y_{0} \rightarrow X_{0}$ be a log-resolution with exceptional set $E \subseteq Y_{0}$ and let $X=X_{0} \times C$. In this case $Y_{0} \times C \rightarrow X$ is a log-resolution with exceptional set $E \times C$. We have the following:

$$
h_{s t}^{2,2}(X)=h_{s t}^{2,2}\left(X_{0}\right)+h_{s t}^{2,1}\left(X_{0}\right) h^{0,1}(C)+h_{s t}^{1,2}\left(X_{0}\right) h^{1,0}(C)+h_{s t}^{1,1}\left(X_{0}\right) .
$$

If $E_{s t}\left(X_{0}\right)$ is a polynomial, then $h_{s t}^{2,2}\left(X_{0}\right)=h_{s t}^{1,1}\left(X_{0}\right) \geq 0$ by Theorem B. This implies that $h_{s t}^{2,2}(X) \geq 0$ by applying Theorem B to each of the stringy Hodge numbers in the expression. But even if it is not a polynomial we can get the same conclusion by applying Corollary $\mathrm{C}$, since it implies

we obtain as above

$$
h_{s t}^{2,2}\left(X_{0}\right) \geq 0,
$$

$$
h_{s t}^{2,2}(X) \geq 0 .
$$

12.2. We describe next a class of terminal fourfolds $X$ that admit a log-resolution which satisfies condition $(*)$.

For the purpose of this paper we introduce the following terminology.

Definition 12.2. Let $X$ be a fourfold with Gorenstein terminal singularities. Let $C \subseteq X$ be an irreducible curve in the singular locus of $X$. We say that $X$ is equisingular along $C$ if it is locally a hypersurface in $\mathbb{C}^{5}$, say with coordinates $x, y, z, w, t$, and $C$ is locally a complete intersection given by $(x=y=z=w=0)$, such that, outside a finite set of points, the singularities in the hyperplanes $t=a \in \mathbb{C}$ are analytically isomorphic. We say that $X$ is strongly equisingular along $C$ if it is equisingular and the condition is satisfied on every point of $C$.

Definition 12.3. We say that a singularity of a terminal threefold admits a controlled resolution if it admits a log-resolution of singularities such that:

(i) it consists of a sequence of blow-ups of points, such that at each step the exceptional divisor has only isolated cDV singularities ${ }^{3}$ which do not have the same cDV type or do not have the same Milnor number,

(ii) the double intersections of the irreducible components of the exceptional divisor are connected.

We say that it admits a strong controlled resolution if admits a controlled one, such that after each blow-up the exceptional divisor has a unique singular point.

Proposition 12.4. Let $X$ be a fourfold with Gorenstein terminal singularities and let $C$ be the one-dimensional singular locus. Suppose that one of the following is true:

(i) For every irreducible component of $C, X$ is equisingular along it and the singularity on the hyperplane sections admits a strong controlled resolution.

(ii) Every connected component of $C$ is irreducible, $X$ is strongly equisingular along it, and the singularity on the hyperplane sections admits a controlled resolution.

\footnotetext{
${ }^{3} \mathrm{~A}$ better approach would perhaps be, instead of taking a sequence of regular blow ups, to take explicit resolutions in the sense of Chen, which consist of a sequence of weighted blow ups [Che16].
} 
Then $X$ admits a log-resolution which satisfies condition $(*)$.

Proof. We can restrict to one of the irreducible components. For (i), we can consider the intersection points with other components as points on which $X$ is not equisingular along $C$, as this does not affect the fibers. From now on we assume $C$ is irreducible.

Let $X_{0}$ be an analytic threefold with an isolated singularity of the type determined by $C$, and

$$
Y_{0}=X_{0}^{n} \rightarrow X_{0}^{n-1} \rightarrow \cdots \rightarrow X_{0}^{1} \rightarrow X_{0}
$$

be the sequence of blowups of the (strong) controlled resolution. We can assume $X_{0}^{1} \rightarrow X_{0}$ is the blowup of the point. Let

$$
X^{1} \rightarrow X
$$

be the blow up of $X$ along $C$. Let $U \subseteq X$ be one open set which makes $X$ satisfy the condition of equisingularity (see Definition 12.2). In this open set, we are taking the blow up of the ideal $(x, y, z, w)$ and we denote the blow up by $U^{1}$. Let $U_{k}$ be the subvarieties in $U$ defined by $t=k \in \mathbb{C}$. In each of the $U_{k}$ we are taking the blow up along the only singular point, denoted by $U_{k}^{1}$. As $U_{k} \cong X_{0}$, the first blowup is isomorphic to $X_{0}^{1}$. The next step $X_{0}^{2} \rightarrow X_{0}^{1}$ is a blow up along a subvariety of $X_{0}^{1}$, which determines a subvariety on $U_{k}^{1}$. Taking the same ideal on $U^{1}$, gives a subvariety such that when restricted to $U_{k}^{1}$ was the original one. We define

$$
U^{2} \rightarrow U^{1}
$$

to be the blow up along that subvariety.

The blow up is well defined. Indeed, let $V \subseteq X$ be a different open set such that $U \cap V \neq \emptyset$, and $V^{1} \rightarrow V$ the first blowup. For (i), as the resolution of $X_{0}$ is strongly controlled, the subvariety determined by $V^{1}$ and $U^{1}$ on $V^{1} \cap U^{1}$ agree, as is just the new singular locus on each hyperplane section. For (ii), each of the isolated singularities in the $U_{k}^{1}$ must coincide with the corresponding singularities in the subvarieties of $V^{1}$. Indeed, on an analytic open set the cDV type of the singularity is the same (see [Nam01]), and outside of finite points, the Milnor number is constant in an open set around the point (see [GLS07, Proposition 2.57]). The strongly equisingular condition ensures that the subvariety we blow up in the next step has different connected components corresponding to different singularities we get in each fiber, and in particular we get a global subvariety that is being blown up.

By using the same argument as above on every step of the (strong) controlled resolution, we get a birational morphism

$$
Y^{\prime}=X^{n} \rightarrow X^{n-1} \rightarrow \cdots \rightarrow X^{1} \rightarrow X
$$

such that $Y^{\prime}$ only has singularities whose image in $X$ is a finite set of points. Let $Y \rightarrow Y^{\prime}$ be a log-resolution of singularities which is an isomorphism outside of the singular locus of $Y^{\prime}$. To check that the conditions of $(*)$ are satisfied on $Y$, is enough to check them on $Y^{\prime}$. The exceptional divisors in $Y^{\prime}$ come from the irreducible components being blown up during the process. Therefore, to each of these corresponds one of the divisors in $Y_{0}$. The double intersections in $Y^{\prime}$ correspond to double intersections in $Y_{0}$ by the condition (ii) of controlled resolutions. Therefore the resolution satisfies $(*)$. 
Example 12.5. For certain types of singularities of terminal threefolds explicit resolutions have been constructed. For instance, it can be checked that singularities of type $A_{n}$ and $E_{6}$ admit strong controlled resolutions and those of type $D_{2 k+1}$ admit controlled resolutions (see [DR01]).

There are also some examples coming from the MMP of varieties for which Proposition 12.4 can be applied. In [Tak99] and [AW98] the authors study the singularities of an extremal contraction of a smooth fourfold. Let $Z$ be a smooth fourfold and $h: Z \rightarrow X$ a contraction of a extremal ray. Assume that the contraction is of type $(3,1)$, that is, the exceptional set is a divisor and is contracted to dimension 1. A complete classification is given: let $E$ be the exceptional divisor of $h$ and consider $\left.h\right|_{E}: E \rightarrow C$. This map is either a $\mathbb{P}^{2}$-bundle or a quadric bundle. In the case of a $\mathbb{P}^{2}$-bundle the variety is smooth or the singularities are not of index 1 . In the other cases $X$ have as singular locus the smooth curve $C$. In the list of possible local equations, we can verify that they satisfy the equisingularity condition involving the singularities $A_{1}$ and $A_{2}$. Note that in the case of $A_{2}$, the map $h$ is not a log-resolution.

\section{REFERENCES}

[AW98] M. Andreatta and J. A. Wiśniewski, Contractions of smooth varieties. II. Computations and applications, Boll. Unione Mat. Ital. Sez. B Artic. Ric. Mat. (8) 1 (1998), no. 2, 343-360. MR1638131

[Bat98] V. V. Batyrev, Stringy Hodge numbers of varieties with Gorenstein canonical singularities, Integrable systems and algebraic geometry (Kobe/Kyoto, 1997), 1998, pp. 1-32. MR1672108

[Bat99] _ Non-Archimedean integrals and stringy Euler numbers of log-terminal pairs, J. Eur. Math. Soc. (JEMS) 1 (1999), no. 1, 5-33. MR1677693

[BCHM10] C. Birkar, P. Cascini, C. D. Hacon, and J. McKernan, Existence of minimal models for varieties of log general type, J. Amer. Math. Soc. 23 (2010), no. 2, 405-468. MR2601039

[Car53] H. Cartan, Variétés analytiques complexes et cohomologie, Colloque sur les fonctions de plusieurs variables, tenu à Bruxelles, 1953, 1953, pp. 41-55. MR0064154

[CEZGL14] E. Cattani, F. El Zein, P. A. Griffiths, and D. T. Lê (eds.), Hodge theory, Mathematical Notes, vol. 49, Princeton University Press, Princeton, NJ, 2014. MR3288678

[Che16] J. A. Chen, Explicit resolution of three dimensional terminal singularities, Minimal models and extremal rays (Kyoto, 2011), 2016, pp. 323-360. MR3618265

[dCM05] M. A. A. de Cataldo and L. Migliorini, The Hodge theory of algebraic maps, Ann. Sci. École Norm. Sup. (4) 38 (2005), no. 5, 693-750. MR2195257

[dCM07] M. A. de Cataldo and L. Migliorini, Intersection forms, topology of maps and motivic decomposition for resolutions of threefolds, Algebraic cycles and motives. Vol. 1, 2007, pp. 102-137. MR2385301 (2009a:14009)

[Del71] P. Deligne, Théorie de Hodge. II, Inst. Hautes Études Sci. Publ. Math. 40 (1971), 5-57.

[Del74] _ Théorie de Hodge. III, Inst. Hautes Études Sci. Publ. Math. 44 (1974), 5-77.

[DR01] D. I. Dais and M. Roczen, On the string-theoretic Euler numbers of 3-dimensional A-D-E singularities, Adv. Geom. 1 (2001), no. 4, 373-426. MR1881747

[Elz83] F. Elzein, Mixed Hodge structures, Singularities, Part 1 (Arcata, Calif., 1981), 1983, pp. 345-352. MR713073

[GKKP11] D. Greb, S. Kebekus, S. J. Kovács, and T. Peternell, Differential forms on log canonical spaces, Publ. Math. Inst. Hautes Études Sci. 114 (2011), 87-169. MR2854859

[GLS07] G.-M. Greuel, C. Lossen, and E. Shustin, Introduction to singularities and deformations, Springer Monographs in Mathematics, Springer, Berlin, 2007. MR2290112

[GM88] M. Goresky and R. MacPherson, Stratified Morse theory, Ergebnisse der Mathematik und ihrer Grenzgebiete (3) [Results in Mathematics and Related Areas (3)], vol. 14, Springer-Verlag, Berlin, 1988. MR932724 
[HTT08] R. Hotta, K. Takeuchi, and T. Tanisaki, D-modules, perverse sheaves, and representation theory, Progress in Mathematics, vol. 236, Birkhäuser Boston, Inc., Boston, MA, 2008. Translated from the 1995 Japanese edition by Takeuchi. MR2357361

[HW01] J. W. Hoffman and S. H. Weintraub, The Siegel modular variety of degree two and level three, Trans. Amer. Math. Soc. 353 (2001), no. 8, 3267-3305. MR1828606

[Kaw88] Y. Kawamata, Crepant blowing-up of 3-dimensional canonical singularities and its application to degenerations of surfaces, Ann. of Math. (2) 127 (1988), no. 1, 93-163. MR924674

[KM98] J. Kollár and S. Mori, Birational geometry of algebraic varieties, Cambridge Tracts in Mathematics, vol. 134, Cambridge University Press, Cambridge, 1998. With the collaboration of C. H. Clemens and A. Corti, Translated from the 1998 Japanese original. MR1658959

[MP05] M. Mustaţă and S. Payne, Ehrhart polynomials and stringy Betti numbers, Math. Ann. 333 (2005), no. 4, 787-795. MR2195143

[Nam01] Y. Namikawa, A note on symplectic singularities, ArXiv Mathematics e-prints (January 2001), available at math/0101028.

[NS95] Y. Namikawa and J. H. M. Steenbrink, Global smoothing of Calabi-Yau threefolds, Invent. Math. 122 (1995), no. 2, 403-419. MR1358982

[PS08] C. A. M. Peters and J. H. M. Steenbrink, Mixed Hodge structures, Ergebnisse der Mathematik und ihrer Grenzgebiete. 3. Folge. A Series of Modern Surveys in Mathematics [Results in Mathematics and Related Areas. 3rd Series. A Series of Modern Surveys in Mathematics], vol. 52, Springer-Verlag, Berlin, 2008. MR2393625 (2009c:14018)

[Rei80] M. Reid, Canonical 3-folds, Journées de Géometrie Algébrique d'Angers, Juillet 1979/Algebraic Geometry, Angers, 1979, 1980, pp. 273-310. MR605348

[Rei83] _ Minimal models of canonical 3-folds, Algebraic varieties and analytic varieties (Tokyo, 1981), 1983, pp. 131-180. MR715649

[Sch06] J. Schepers, Stringy E-functions of varieties with A-D-E singularities, Manuscripta Math. 119 (2006), no. 2, 129-157. MR2215965

[Sch12] Stringy Hodge numbers of strictly canonical nondegenerate singularities, J. Algebraic Geom. 21 (2012), no. 2, 273-297. MR2877435

[Ste83] J. H. M. Steenbrink, Mixed Hodge structures associated with isolated singularities, Singularities, Part 2 (Arcata, Calif., 1981), 1983, pp. 513-536. MR713277

[SV07] J. Schepers and W. Veys, Stringy Hodge numbers for a class of isolated singularities and for threefolds, Int. Math. Res. Not. IMRN 2 (2007), Art. ID rnm016, 14. MR2361454 (2009e:32033)

[Tak99] H. Takagi, Classification of extremal contractions from smooth fourfolds of $(3,1)$-type, Proc. Amer. Math. Soc. 127 (1999), no. 2, 315-321. MR1637436

[Voi07] C. Voisin, Hodge theory and complex algebraic geometry. I, English, Cambridge Studies in Advanced Mathematics, vol. 76, Cambridge University Press, Cambridge, 2007. Translated from the French by Leila Schneps. MR2451566

[Yas04] T. Yasuda, Twisted jets, motivic measures and orbifold cohomology, Compos. Math. 140 (2004), no. 2, 396-422. MR2027195 USA

E-mail address: seolano@math.northwestern.edu 